\title{
Observations of plasma density structures in association with the passage of traveling convection vortices and the occurrence of large plasma jets
}

\author{
C. E. Valladares ${ }^{1}$, D. Alcaydé ${ }^{2}$, J. V. Rodriguez ${ }^{3}$, J. M. Ruohoniemi ${ }^{4}$, A. P. Van Eyken \\ ${ }^{1}$ Institute for Scientific Research, Boston College, Newton Center, MA, USA \\ ${ }^{2}$ CESR-CNRS - 9 Av. Cl Roche - F31028 Toulouse Cedex 04 - France \\ ${ }^{3}$ Ball Aerospace \& Technologies Corp., Boulder, CO, USA \\ ${ }^{4}$ The Johns Hopkins University, Applied Physics Lab, Laurel, MD, USA \\ ${ }^{5}$ EISCAT Scientific Association, Ramfjordmoen, N9020 Tromsdalen, Norway
}

Received: 16 February 1998 / Revised: 23 November 1998 / Accepted: 26 November 1998

\begin{abstract}
We report important results of the first campaign specially designed to observe the formation and the initial convection of polar cap patches. The principal instrumentation used in the experiments comprised the EISCAT, the Sondrestrom, and the Super DARN network of radars. The experiment was conducted on February 18, 1996 and was complemented with additional sensors such as the Greenland chain of

changes in the LPJs location is to create regions containing dayside plasma almost detached from the rest of the oval density. One of these density features was seen by the Sondrestrom radar at 1542 UT. The data presented here suggest that two plasma structuring mechanisms (TCVs and LPJs) can act tens of minutes apart to produce higher levels of density structures in the near noon F-region ionosphere.
\end{abstract} magnetometers and the WIND and IMP-8 satellites. Two different types of events were seen on this day, and in both events the Sondrestrom radar registered the formation and evolution of large-scale density structures. The first event consisted of the passage of traveling convection vortices (TCV). The other event occurred in association with the development of large plasma jets (LPJ) embedded in the sunward convection part of the dusk cell. TCVs were measured, principally, with the magnetometers located in Greenland, but were also confirmed by the line-of-sight velocities from the Sondrestrom and SuperDARN radars. We found that when the magnetic perturbations associated with the TCVs were larger than $100 \mathrm{nT}$, then a section of the high-latitude plasma density was eroded by a factor of 2 . We suggest that the number density reduction was caused by an enhancement in the $\mathrm{O}^{+}$recombination due to an elevated $\mathrm{T}_{\mathrm{i}}$, which was produced by the much higher frictional heating inside the vortex. The large plasma jets had a considerable $(>1000 \mathrm{~km})$ longitudinal extension and were $200-300 \mathrm{~km}$ in width. They were seen principally with the Sondrestrom, and SuperDARN radars. Enhanced ion temperature $\left(T_{i}\right)$ was also observed by the Sondrestrom and EISCAT radars. These channels of high $T_{i}$ were exactly collocated with the LPJs and some of them with regions of eroded plasma number density. We suggest that the LPJs bring less dense plasma from later local times. However, the recent time history of the plasma flow is important to define the depth of the density depletion. Systematic changes in the latitudinal location and in the intensity of the LPJs were observed in the 2 min time resolution data of the SuperDARN radars. The effect of the abrupt
Key words. Ionosphere (ionospheric irregularities) · Magnetospheric physics (electric fields; polar cap phenomena)

\section{Introduction}

Since the first observations of plasma density were conducted at very high latitudes, it became evident that large-scale $(>100 \mathrm{~km})$ plasma density structures were always present within the polar cap (Hill, 1963; Sato and Rourke, 1964). The first systematic examination of these polar cap structures started years later when several complementary instruments, such as an all-sky intensified photometer (ASIP), a digital ionosonde, and scintillation receivers operated simultaneously at Thule, Greenland ( $86^{\circ}$ geomagnetic latitude) (Buchau et al., 1983). Soon thereafter, it was concluded that two important classes of large-scale density structures prevailed within the polar cap: sun-aligned arcs and polar cap patches (Weber and Buchau, 1981).

The works by Lassen (1972), Berkey et al. (1976) and Ismail et al. (1977) had previously demonstrated that the sun-aligned F-region arcs existed inside the polar cap preferentially when the magnetic conditions were quiet or equivalently when the $B_{z}$ component of the IMF was directed northward. In analogy to this discovery, Buchau et al. (1983) noted that the polar cap patches 
occurred during magnetically active periods or IMF $B_{z}$ southward conditions. Buchau et al. (1983) defined the patches as spatially confined enhancements in the Fregion number density above its background level. The density enhancements are usually a factor of 2 during solar minimum conditions, but can reach a factor of 10 during solar maximum years (Buchau and Reinisch, 1991). The patches move in the antisunward direction (Fukui et al., 1994), lack polar cap particle precipitation (excluding polar rain) (Weber et al., 1986), have sizes between 100 and $1000 \mathrm{~km}$, and possess a patchy signature in the $630.0 \mathrm{~nm}$ airglow emissions.

The strong UT dependence of the patch occurrence in the Northern Hemisphere, and the fact that the auroral oval, almost fixed in magnetic coordinates, is able to reach lower geographic latitudes at the same UT hours that the patches are observed, provide strong support for the source of the high density plasma of the patches to be sun-produced plasma created at latitudes equatorward or near the auroral oval (Buchau et al., 1985; de la Beaujardiere et al., 1985). Incoherent scatter radar observations by Foster and Doupnik (1984) and by Kelly and Vickrey (1984) in fact confirmed that subauroral/auroral plasma gets routed through the dayside throat region and enters into the polar cap forming a continuous and elongated tongue-of-ionization (TOI). While the observation of the TOI, extending all the way across the polar cap, provided a source of the enhanced plasma, the patchy nature of the density enhancements implied the existence of an additional mechanism capable of breaking off the seemingly continuous TOI.

Several mechanisms have been suggested to explain the formation of polar cap patches and their complete detachment from the auroral oval (see reviews by Tsunoda, 1988 and Crowley, 1996 for a general discussion of formation mechanisms). In a general sense, the formation of polar cap patches could be explained by the particular characteristics of the plasma electrodynamics that occurs in the midday cusp region (Rodger and Graham, 1996). Therefore, it is worthwhile to observe in great detail all the physical processes that occur near the midday ionosphere. Two prominent processes in this region are the large flows and the midday auroral breakup events that have been observed with the EISCAT incoherent scatter radar (ISR) in association with cusp dynamics (Sandholt et al., 1990; Lockwood et al., 1990). The large plasma flows have been explained by Cowley et al. (1991) to form channels in the region adjacent and poleward of the open-closed field line boundary. These large flows are a direct result of the tension force on newly opened field lines. The role of the large plasma flows in the formation of patches has, in fact, been introduced by Rodger et al. (1994). These authors, based on ionospheric velocities measured by the PACE radar, concluded that short-lived plasma jets $(\sim 2 \mathrm{~km} / \mathrm{s})$ occurred within a few minutes of the formation of polar cap patches and near the dayside cusp. Additional support for the large flow mechanism was provided by Valladares et al. (1994). Measurements conducted with the Sondrestrom ISR showed that the large flows were exactly collocated with density deple- tions and elevated ion temperatures. It was argued then that enhanced $\mathrm{O}^{+}$recombination, due to the elevated $\mathrm{T}_{\mathrm{i}}$ values, eroded a section of the TOI and created a discrete entity of high density plasma. A modeling effort of this event, using a set of twin vortices to represent the large plasma flow, showed that the detachment of the patch from the auroral oval occurs not when the twin vortices are stationary, but instead when they are allowed to move with the background plasma (Valladares et al., 1996). These authors also demonstrated that even for vortices extending $9^{\circ}$ in longitude and with a $25 \mathrm{kV}$ potential drop, $60 \%$ of the plasma density decrease can be attributed to transport of less dense plasma from earlier or later local times.

The purposes of this work are: to present the variability of the plasma velocity structures in a much larger spatial context and with higher temporal resolution than has been presented before, to provide the longitudinal extension of the large plasma flows, and to evaluate the uniformity of the plasma density in regions away from the convection throat region. Another motivation consists of discussing how these velocity features act to produce density break-off events and therefore increase the level of plasma density structuring.

With these goals in mind, a major campaign was launched on February 1996 with the participation of the EISCAT and the Sondrestrom incoherent scatter radars (ISR) and the Super-DARN network of coherent radars. This study presents data that was collected on February 18, 1996, during this campaign. The events described here occurred after the IMF $B_{z}$ reversed to a southward direction. While the general goals of the project were to observe how the patches are formed and how they exit from the polar cap, in this publication we only deal with the formation of polar cap patches and their entrance into the polar cap.

\section{General observations}

As mentioned in the introduction, the following highlatitude radars provided precise information on the ionospheric parameters: the EISCAT, Tromso ISR, the Sondrestrom, Greenland ISR, and several HF coherent radars of the SuperDARN network (Greenwald et al., 1995). In addition, the magnetic field values measured by the Greenland chain of magnetometers were also included into this study. Figure 1 shows the location and the area probed by both incoherent scatter radars. As indicated in this figure, the Sondrestrom radar conducted a series of $360^{\circ}$ azimuth scans followed by an elevation scan in a plane perpendicular to a constant invariant latitude line ( $-27^{\circ}$ from geographic north). A sequence of an azimuth and an elevation scans was repeated every $8 \mathrm{~min}$. The EISCAT UHF radar operated in an azimuth scanning mode, covering an area of $90^{\circ}$. The scans started at geographic north and reached geographic west in $15 \mathrm{~min}$. This was one of the first opportunities that such a type of scan was exercised with the EISCAT UHF radar. The EISCAT VHF antenna beam was split in two directions to provide a measure of 


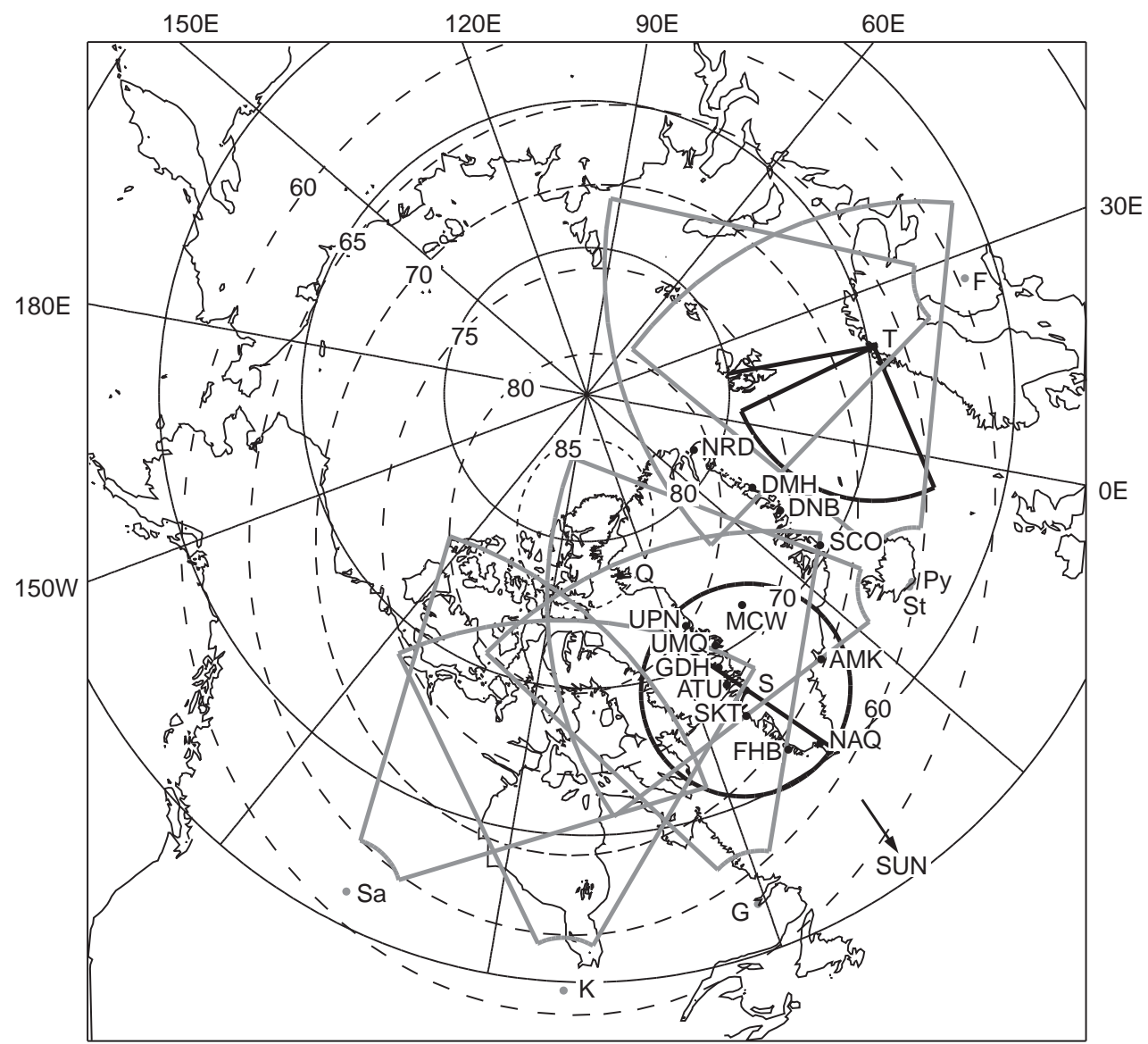

Fig. 1. Illustration of the different radar sites and their approximate fields-of-view (FOV) located at high latitudes. The gray traces delineate the areas probed by the SuperDARN radars that were operational during the experiment. The light gray contour represents the FOV of the Goose Bay radar. The antenna-scanning configuration of the Sondrestrom and EISCAT radars used on February 18, 1996, is displayed in black. The large black dots indicate the locations where the radars and magnetometers of the Greenland chain are placed. Their geographic locations are as follow: $\mathrm{Q}=$ Qaanaaq $\left(77.5^{\circ} \mathrm{N}, \quad 69.2^{\circ} \mathrm{W}\right) ; \quad S=$ Sondrestrom $\left(66.9^{\circ} \mathrm{N}, \quad 50.9^{\circ} \mathrm{W}\right)$; $T=$ Tromso $\left(69.6^{\circ} \mathrm{N}, 19.2^{\circ} \mathrm{E}\right) ; S a=$ Saskatoon $\left(52.2^{\circ} \mathrm{N}, 106.5^{\circ}\right.$ $\mathrm{W}) ; K=$ Kaspuskasing $\left(49.4^{\circ} \mathrm{N}, 82.3^{\circ} \mathrm{W}\right) ; G=$ Goose Bay $\left(53.3^{\circ}\right.$ $\left.\mathrm{N}, 60.5^{\circ} \mathrm{W}\right) ; P y=$ Pykkvibaer $\left(63.8^{\circ} \mathrm{N}, 19.2^{\circ} \mathrm{W}\right) ; F=$ Finland $\left(62.3^{\circ} \mathrm{N}, 26.6^{\circ} \mathrm{E}\right)$ the vector velocity. During the experiment only five of the SuperDARN radars were in operation. Their fieldof-views are depicted by the gray contours of Fig. 1. The area covered by the Goose Bay $(\mathrm{G})$ radar is displayed in light gray to indicate that on February 18, 1996 this radar was on maintenance and did not perform measurement of the plasma velocities. Consequently, we could not combine the line-of-sight velocities from Stokkseyri and Goose Bay to estimate the 2-D horizontal velocity vector. Each of the coherent radars completed a $\sim 60^{\circ}$ scan every 2 min.

\subsection{Solar wind observations}

On February 18, 1996, both the WIND and IMP-8 satellites were favorably located for measuring the IMF. WIND was positioned at $X=+173.8 R_{E}$ distance in front of the magnetopause, and IMP-8 was situated $\left(X=4.5, Y=29.3\right.$, and $\left.Z=28.0 R_{E}\right)$ at the dusk flank of the magnetosphere, and upstream from the bow shock. Figure 2 shows the value of the three compo- nents of the IMF measured by the WIND satellite. This figure shows that between 1330 and 1400 UT the $B_{z}$ IMF component slowly changed from north to south. At $1356 \mathrm{UT}, B_{z}$ becomes negative and remains around $-2 \mathrm{nT}$ for the next $3 \mathrm{~h}$. During the time of the $B_{z}$ reversal, the $B_{y}$ and $B_{x}$ components were near zero and $+5 \mathrm{nT}$ respectively. Thus, at the time of the $B_{z}$ reversal the IMF was practically directed in the $+B_{x}$ direction.

We have estimated the propagation delay for the effect of the IMF transitions, detected by the WIND satellite at $+173 R_{E}$, to be felt at the ionosphere. This calculation is based on the expressions advanced by Lockwood et al. (1989) and the solar wind parameters measured by the WIND and IMP-8 satellites. Both WIND and IMP-8 observed that the solar wind velocity was quite steady and equal to $450 \mathrm{~km} / \mathrm{s}$. However, the IMP-8 measurements were interrupted between 1347 UT and 1642 UT, which is the time of interest. Based on the steadiness of the solar wind velocity observed by WIND, and before and after the data gap for IMP-8, we inferred that the solar wind was $450 \mathrm{~km} / \mathrm{s}$ at the time of the $B_{z}$ reversal. 

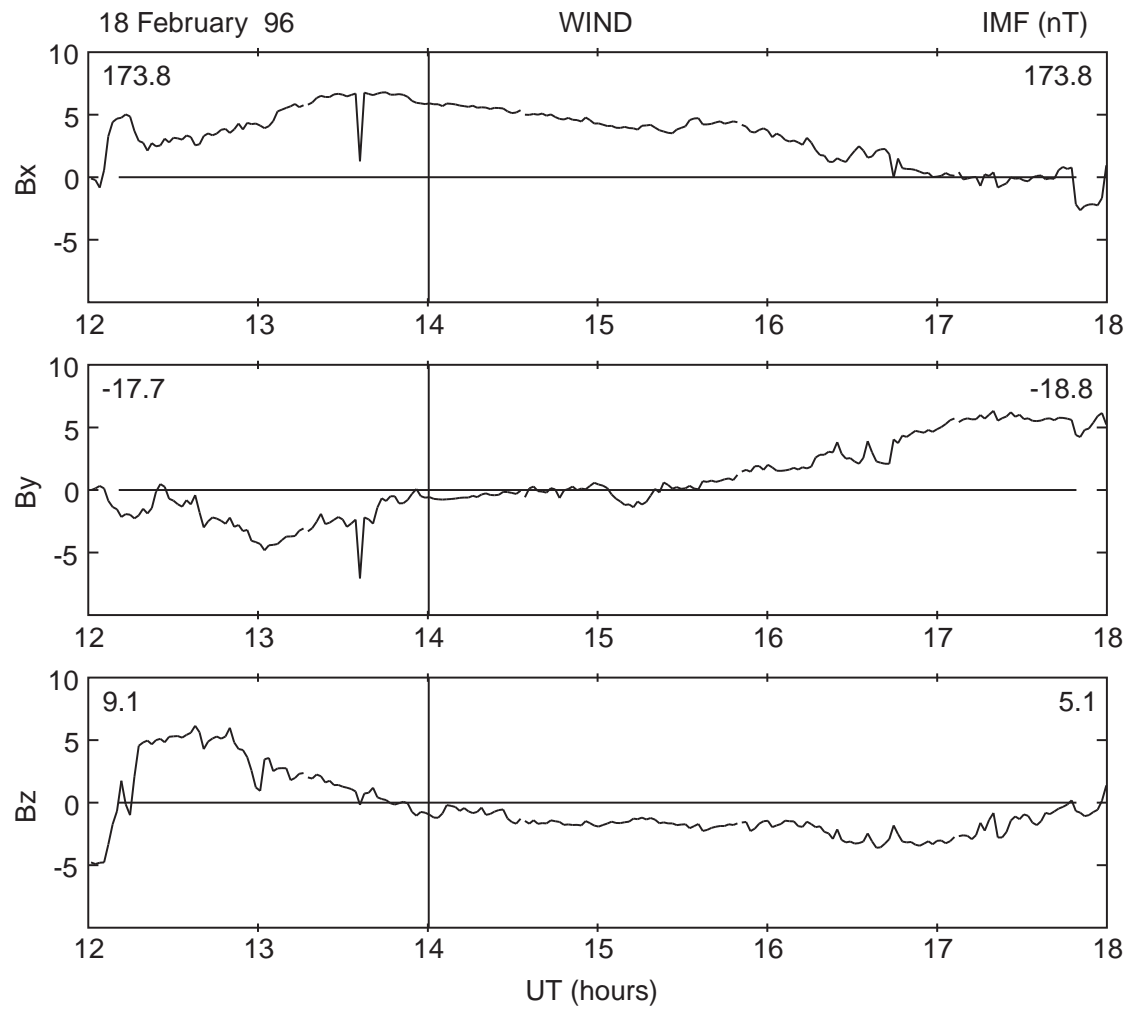

Fig. 2. Interplanetary magnetic field measured by the WIND satellite at 173.8 Earth radii, well in front of the magnetopause. All three IMF components are shown in GSM coordinates. Units for all three quantities are nanoteslas
While the IMF measured by the WIND satellite near the libration point can be in many instances equal to the solar wind magnetic field impinging the magnetopause, it may in some other instances be highly variable in time and space (Kelly et al., 1986). Crooker et al. (1982) have also cautioned that the correlation between the IMF measured by two satellites, one located near the libration point and the other closer to the bow shock, can fall sharply if the satellite positioned toward the Sun is more that $90 R_{E}$ away from the Sun-Earth line. WIND measurements were taken at $Y=+18 R_{E}, Z=+8$ $R_{E}$, less than $90 R_{E}$ from the Sun-Earth line. Thus, we do not expect a drastic variation of the IMF due to the large separation of the spacecraft. To increase the confidence in the IMF values measured by WIND, we performed a correlation analysis between these values and the IMF measured by IMP-8. The agreement was very good for all three IMF components. In fact, a clear delay time of $31 \mathrm{~min}$ was obtained for the three components of the magnetic field gathered near $13 \mathrm{UT}$, and about $40 \mathrm{~min}$ for the IMF values corresponding to 17 UT. Having validated the WIND IMF values, we proceeded to use the expression given by Lockwood et al. (1989), and computed a propagation delay from the WIND satellite location to the cusp equal to $67.7 \mathrm{~min}$.

\subsection{SuperDARN observations}

Figure 3 displays the line-of-sight velocity measured in a sequence of four consecutive scans by five of the SuperDARN HF and VHF radars (Greenwald et al.,
1995). As mentioned earlier only five of the HF radars operated on February 18, 1996. These were: Saskatoon, Kapuskasing, Stokkseyri, Pykkvibaer and Finland radars. Coherent HF radars are sensitive to scattering produced by small-scale F-region number density irregularities. The doppler shift of the backscattered signal is equal to the component of the ionospheric plasma drift along the radar line-of-sight (Ruohoniemi et al., 1987). The SuperDARN velocities, and several other parameters displayed in this work, have been represented employing the corrected geomagnetic coordinate system defined by Baker and Wing (1989). The magnitude and the direction of the velocity have been coded using arrows of different lengths and different types. Figure 3a, corresponding to the observation period between 1506 and 1508 UT, shows the line-of-sight velocity before the passage of a set of TCV. At this time the plasma velocity does not exceed $500 \mathrm{~m} / \mathrm{s}$ in any region where the SuperDARN radars detected coherent echoes. Two minutes later, (Fig. 3b) the velocity in a region near noon suddenly rises to values larger than $1000 \mathrm{~m} / \mathrm{s}$ and the area with irregularities, in the postnoon sector, becomes much broader. A region of large plasma flow extends between $68^{\circ}$ and $80^{\circ}$ magnetic latitude and between 12 and 14 local time. Two channels of large flows seem to form near the dayside. One is centered at $70^{\circ}$ and the other is at $77^{\circ}$ magnetic latitude. Figure $3 \mathrm{c}, \mathrm{d}$ indicates that the large plasma flows persist, at least for the following $4 \mathrm{~min}$, and that the region in between the two channels is filled up, forming a much broader region containing large flows. The spatial distribution of these velocity structures will be defined in Sect. 3 where we 


\section{Super DARN radars}

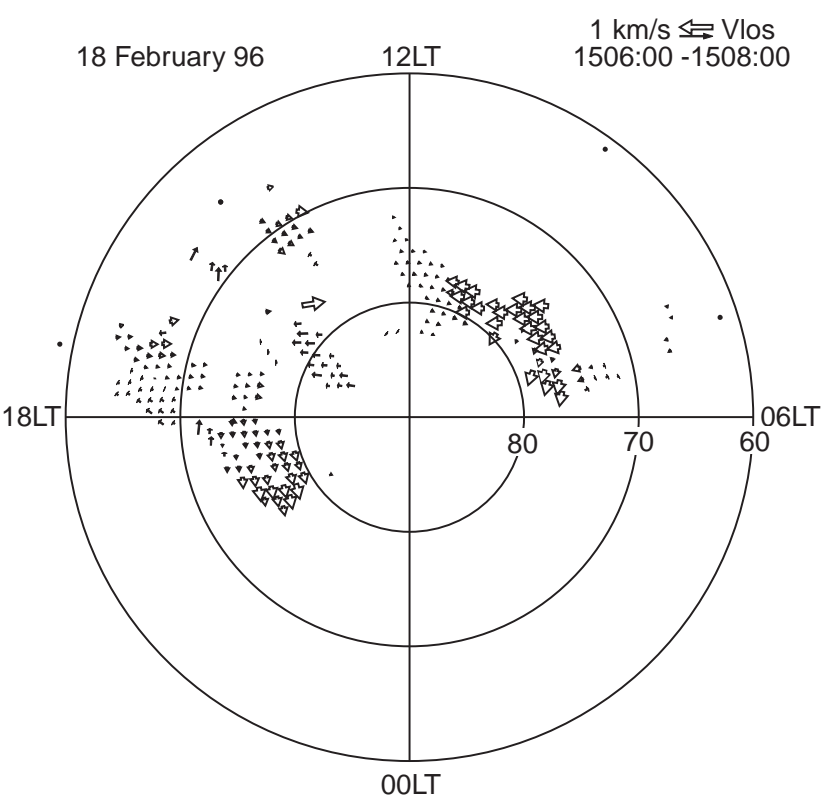

Super DARN radars

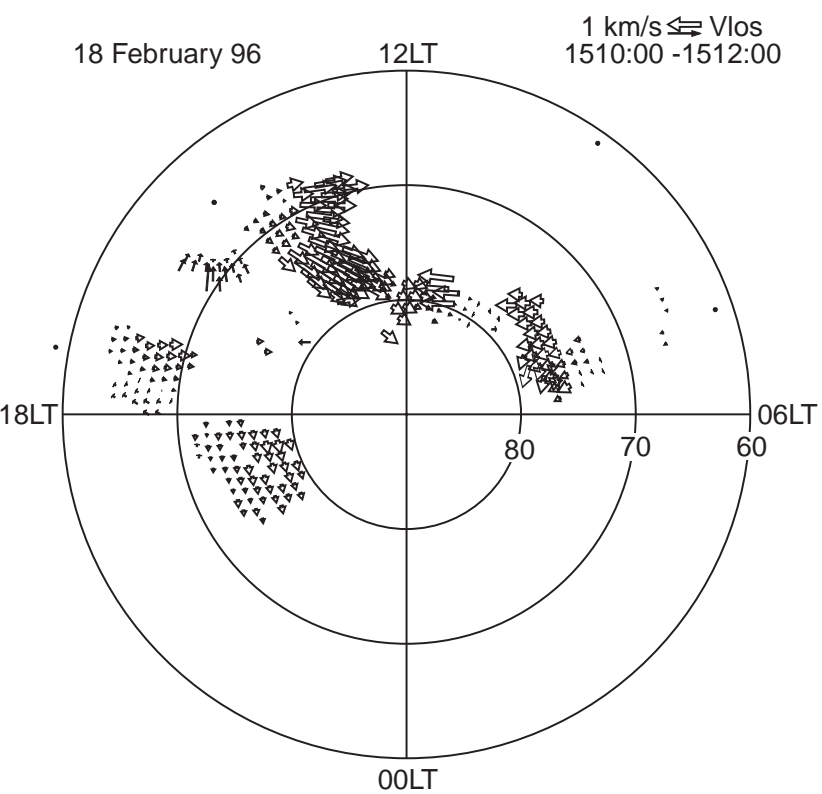

Super DARN radars

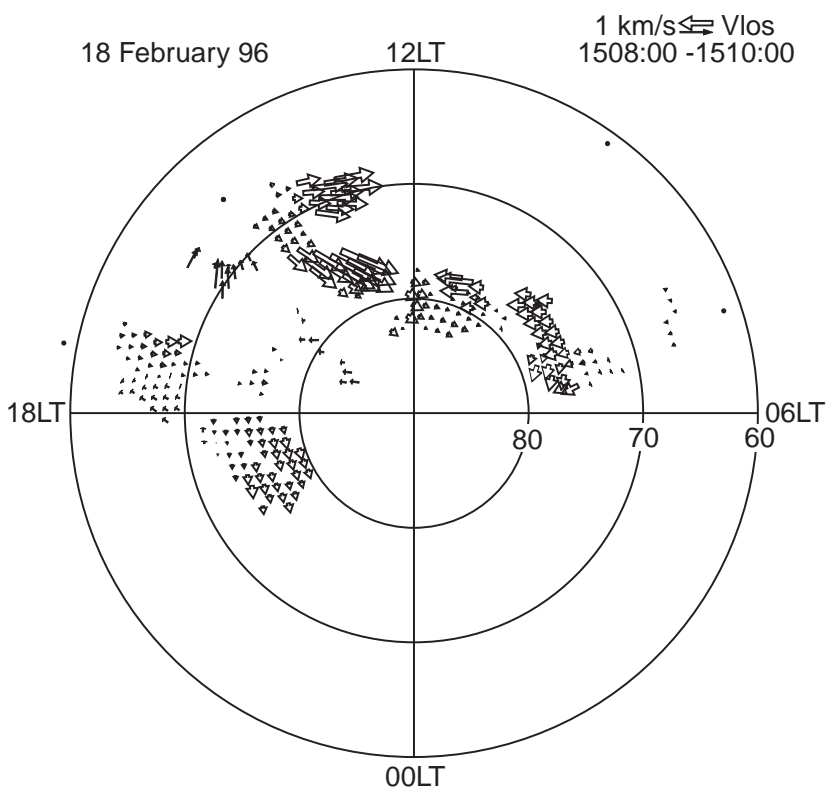

Super DARN radars

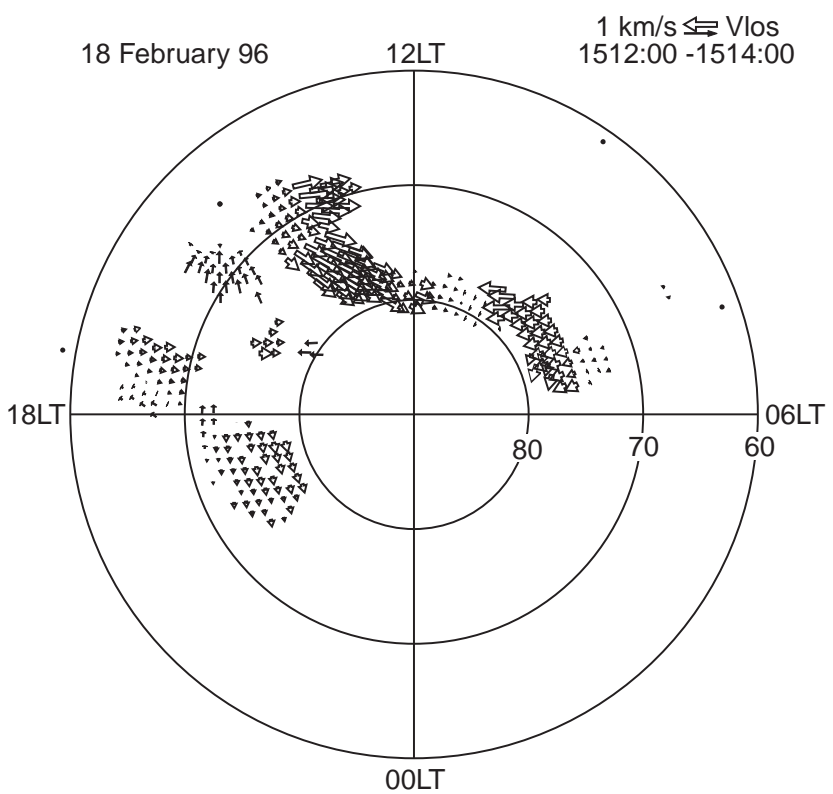

Fig. 3. Line-of-sight velocities measured by five of the SuperDARN radars on February 18, 1996. Note the change in the magnitude of the velocities in the frame that started at $1508 \mathrm{UT}$

combine convection data from different ground-based sensors.

In Fig. 4, we present the cross polar cap potential $\left(\Phi_{\mathrm{PC}}\right)$ that was obtained after conducting a spherical harmonic fitting to all the velocities measured by the HF radars for each of the 2-min segments. Prior to 1506 UT the potential is below $60 \mathrm{kV}$, after this time $\Phi_{\mathrm{PC}}$ presents a series of fluctuations varying between 70 and $90 \mathrm{kV}$. Four $\Phi_{\mathrm{PC}}$ maxima are detected between 1500 and 1630 UT. The first occurs at $1508 \mathrm{UT}$; this is near the time of the initial appearance of the two channels of large plasma flow. We have subtracted $67.7 \mathrm{~min}$ of propaga- tion delay from the time of the first $\Phi_{\mathrm{PC}}$ maximum. The resulting time, 1400:18 UT, is very close to the time when $B_{z}$ reverses sign. This is indicated in Fig. 2 as vertical bars in all three IMF components. The other $\Phi_{\mathrm{PC}}$ maxima occurred at 1530, 1550, and $1606 \mathrm{UT}$. These four polar cap potential intensifications may be attributed to a general increase of the flow velocities within the whole polar cap ionosphere. However, in some cases they could be produced by the sudden appearance of localized velocity structures. A detailed analysis of two of these events is presented in the following two sections. 


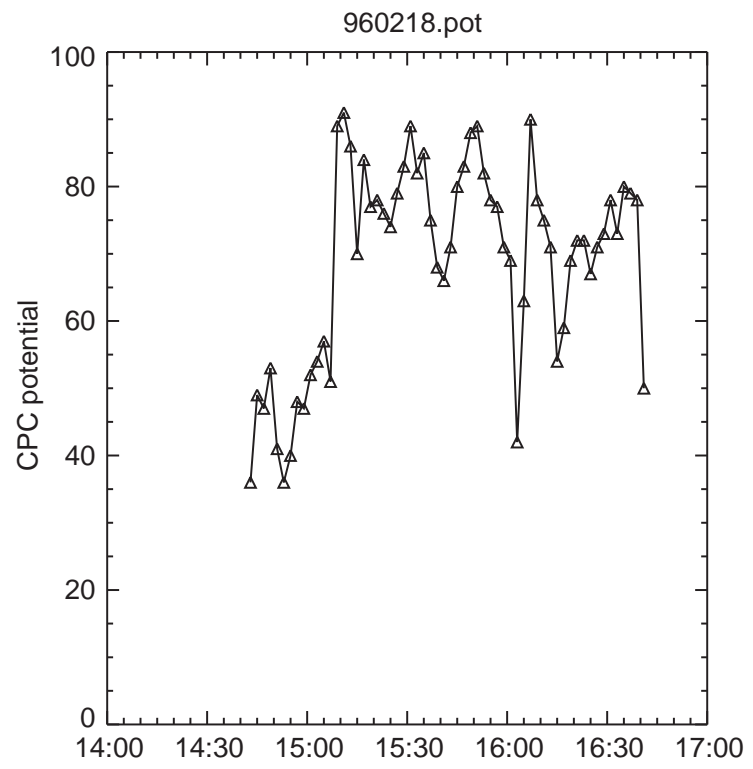

Fig. 4. Time variation of the polar cap potential across the polar cap. The potential was calculated based on the line-of-sight velocity measured by five of the HF SuperDARN radars. Notice the rise of the potential at $1508 \mathrm{UT}$

\section{Patch formation associated with TCV}

The first intensification of the cross polar cap potential (1508 UT) occurred a few minutes after the reversal of $B_{z}$ (including the propagation delay). However, this increase in the polar cap potential seems to be the response of the fitting algorithm to the appearance of two "channels" of large velocities, somewhat localized in their extension and spatially restricted only to the near noon sector.

In this section we combine measurements of magnetic fluctuations gathered by the ground-based magnetometers in Greenland and the line-of-sight velocities from different radars to understand the nature of the velocity structures and the effects that these features produce on the dayside uniform number density. The following subsections deal with an in-depth analysis of the period between 1505 and 1520 UT.

\subsection{Magnetometer observations}

Figure 5 shows 20 -s resolution plots of the magnetic field fluctuations measured by the ground-based magnetometers located on the west coast, ice cap and east coast of Greenland. The seven traces at the top of each panel correspond to stations on the west coast of Greenland. Traces labeled DNB and SCO correspond to stations on the east coast. MCW is one of the MAGIC stations located at the ice cap. The geographic location of these magnetometer stations can be seen in Fig. 1.

The prominent features in Fig. 5 are the large magnetic deviations seen between 1505 and 1530. The D (eastward) and Z (vertical) components show spiky variations with amplitude near $100 \mathrm{nT}$. These magnetic fluctuations resemble the signatures of TCV observed by Friis-Christensen et al. (1988), McHenry et al. [1990], and Clauer and Ridley (1995) also using the Greenland array of magnetometers. Friis-Christensen et al. (1988) argued that their observations were consistent with a twin vortex pattern of convection associated with two field-aligned current filaments separated by $600 \mathrm{~km}$ in the east-west direction. They suggested that the observed TCV were probably related to a readjustment of the magnetopause boundary. More recently, Clauer and Ridley (1995) observed a continuous train of TCVs moving westward across the magnetic noon sector. These authors combined data from the Greenland magnetometers, the Sondrestrom radar and the DMSP satellite to conclude that the source of the vortices was possibly a Kelvin-Helmholtz mechanism or perhaps magnetic merging during an interval of IMF oriented predominantly in the $+B_{y}$ direction. In this publication we are not addressing the association of these TCVs to any magnetospheric/magnetopause process, even though we recognize that this would be a very worthy endeavor. Our goal in this section is to understand the type of $\mathrm{N}_{\mathrm{e}}$ structuring that a passage of a series of TCVs produces in the midday high latitude ionosphere.

\subsection{Sondrestrom ISR observations}

Figure 6 presents maps of the electron number density collected during three consecutive AZ scans and corresponding to the period between 1457:28 and 1519:32 UT. The plot format is similar to Fig. 3 of Valladares et al. (1994), but here the scans cover $360^{\circ}$ of the sky and the antenna elevation angle is $30^{\circ}$. Figure $6 \mathrm{a}$ shows a quasi-uniform F-region, where the F-region peak density slowly decreases from south-to-north following a typical daytime dependency with the SZA. Figure 6b, obtained between 1505:42 and 1511:20 UT, displays narrow density depletion located at the northern part of the scan $(-100,400 \mathrm{~km})$. The time that this $\mathrm{N}_{\mathrm{e}}$ depletion was detected with the Sondrestrom ISR coincides closely with the initiation of the large plasma flows, as measured by the coherent radars, and with the spiky magnetic fluctuations detected by some of the Greenland chain magnetometers. Figure $6 \mathrm{c}$ shows the number densities observed in the following AZ scan that started at 1513:55 UT. This figure reveals that a much broader density depletion was formed in the northeastern region of the scan $(350,350 \mathrm{~km})$. We can not conclude that a density breakoff, or a complete detachment of a plasma slab from the oval has occurred. However, the density plots indicate that the level of structuring has increased. At the same time the density feature has been displaced poleward from a distance of $425 \mathrm{~km}$ from the radar to a location $500 \mathrm{~km}$ away from the radar site. Figure 6d displays a color-coded representation in the Corrected Geomagnetic coordinate system of the number density presented in Fig. 6c.

\subsection{EISCAT ISR observations}

Figure $6 \mathrm{~d}$ shows also the number density measured by the UHF EISCAT ISR during the $90^{\circ}$ azimuth scans 

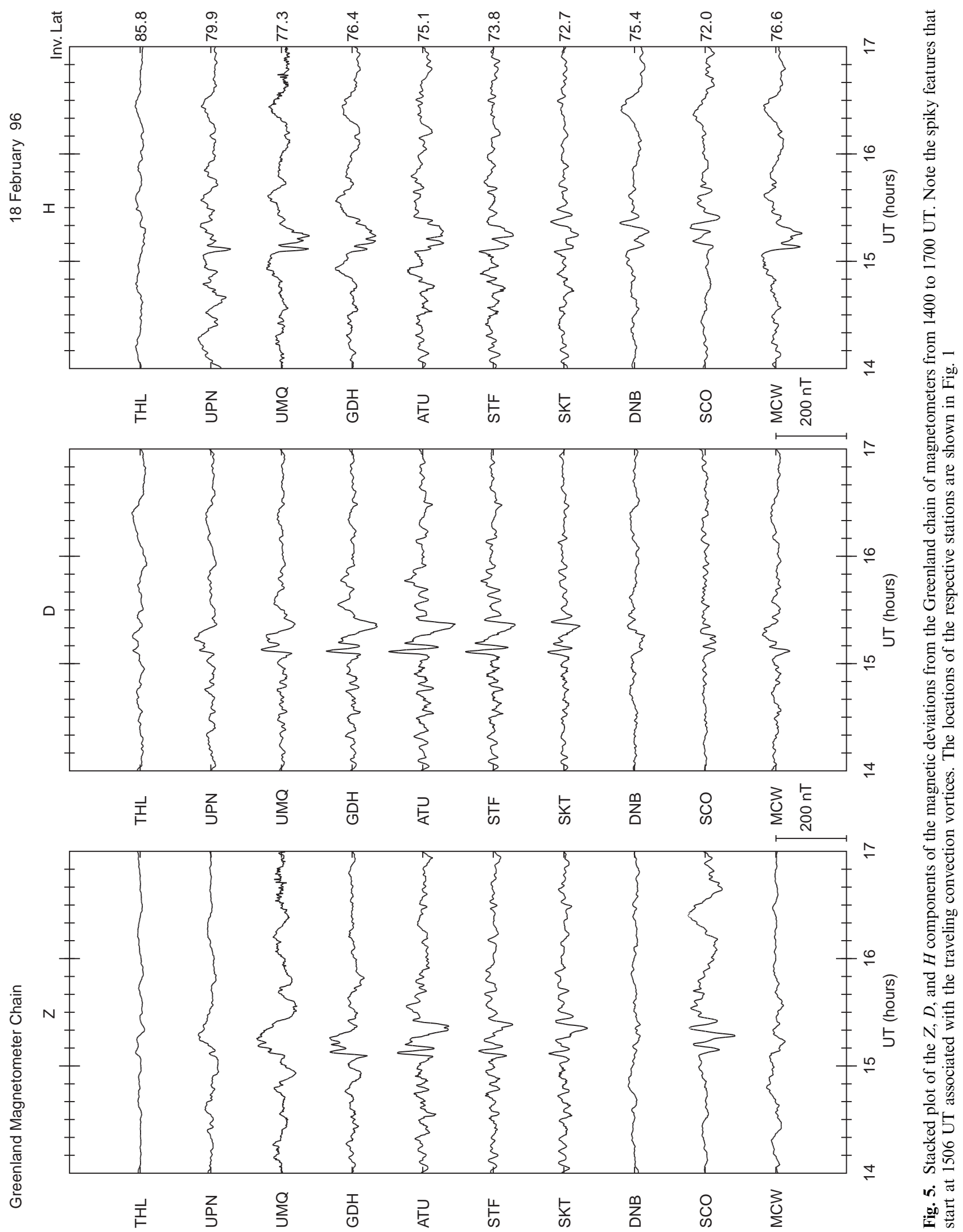

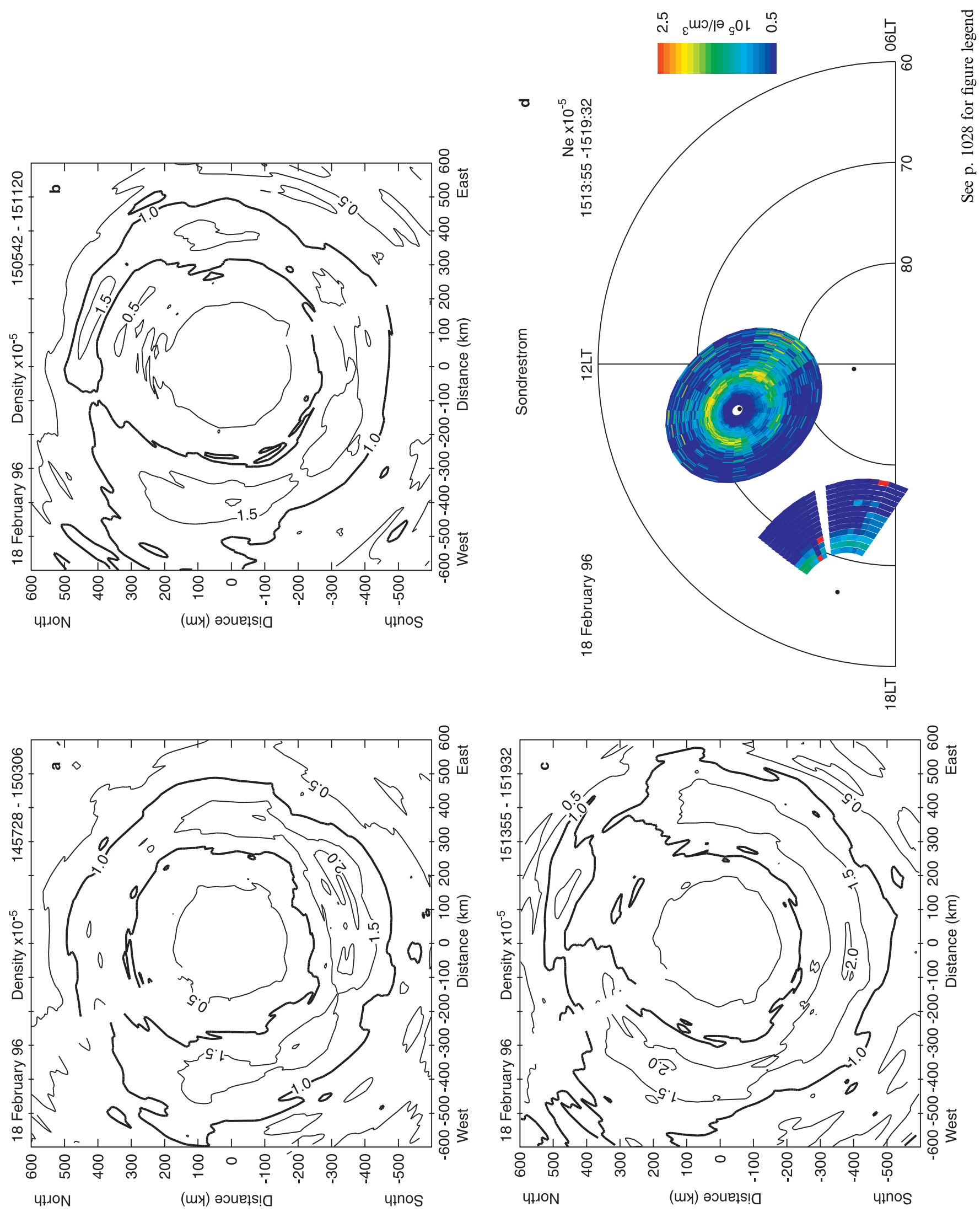
Fig. 6a-d. Plasma density measured by the Sondrestrom ISR on February 18, 1996, during three consecutive azimuth scans. The values gathered during the azimuth scans at $30^{\circ}$ elevation are displayed in the first 3 panels, projected on a plane tangent to the Earth at the radar site. d shows the density measured between 1513:55 and 1519:32 (c) projected into a corrected geomagnetic coordinate system

that were conducted between 1500 and 1515 UT. This partial scan shows that the peak number density is quite uniform and near $1.5 \times 10^{5} \mathrm{~cm}^{-3}$. Neither auroral nor boundary blobs are present in this limited scan. The line-of-sight (LOS) velocities (not shown here) indicated that the area probed by the radar was embedded within a region of sunward convection. The electron temperature was $<2000 \mathrm{~K}$ and uniform suggesting the absence of localized precipitation. Based on the uniform density at EISCAT, we conclude that the structuring seen at Sondrestrom must be created in situ and not transported by the sunward convection.

\subsection{Analysis of localized velocity structures}

In this section, we combine experimental data from different sensors to define the intensity, location and size of the TCVs. Then, we use this information to associate the formation of a deep density depletion, as seen with the Sondrestrom ISR, to the precise location of the TCV containing large flows. Each of the techniques that were used to obtain the plasma drift suffer some inherent limitations. These limitations are in the form of the spatial and temporal coverage and in the relationship between the measured geophysical quantity and the plasma convection. An incoherent scatter radar (and also a coherent radar) provides a direct measurement of the $\mathbf{E} \times \mathbf{B}$ plasma drift. But, these measurements give only one component of the 2-dimensional horizontal flow. [The third component, the one along the B field, is usually assumed to be null. Valladares and Carlson (1991) have reported precise measurements of the plasma velocity component along the field line, undertaken also with the Sondrestrom ISR, of a value equal to $25 \mathrm{~m} / \mathrm{s}$. This value is well below the $50 \mathrm{~m} / \mathrm{s}$ statistical uncertainty of the LOS velocity.] The Sondrestrom ISR employed $5.5 \mathrm{~min}$ to complete a $360^{\circ}$ azimuth scan. The SuperDARN radar used $2 \mathrm{~min}$ to scan a sector close to $60^{\circ}$. These scanning rates highly impair the possibility of a radar to fully probe the velocities associated with a rapidly moving TCV, such as the ones observed on February 18, 1996. The magnetic deflections measured by ground-based magnetometers are closely related to the Hall currents, and these currents are proportional to the plasma convection. Therefore, our analysis of the TCV velocities heavily relies on the magnetic field data.

A cross correlation between the $\mathrm{H}$ component of the magnetic fluctuations from two stations, GDH (west coast) and MCW (MAGIC), located at approximately the same magnetic latitude but $500 \mathrm{~km}$ to the east, gives a phase delay of $87 \mathrm{~s}$. The magnetic pulse first appears at GDH and then travels to MCW. This implies a phase motion of the vortex in the eastward direction equal to $5.7 \mathrm{~km} / \mathrm{s}$.

Figure 7, plotted in a time reversed coordinate, shows the equivalent drifts from the stations located on the west coast of Greenland using the common convection vortex format (Clauer and Ridley, 1995). In this representation we can observe the east-west extension of the vortices. To display the equivalent plasma convection, the magnetic traces were rotated $90^{\circ}$ counter-clockwise, and were also filtered subtracting a $40 \mathrm{~min}$ running average. Several vortices are clearly identified in Fig. 7; a few others are more difficult to interpret due to the rapid evolution of the vortex electrodynamics. The first vortex appears at 1505 UT and corresponds to a clockwise plasma rotation. A second vortex seems to transit at 1508 and a third at 1510 UT. However, the latter two vortices were not completely defined due to their rapid time evolution when they were crossing Greenland's west coast. Figure 8 presents a quasiinstantaneous view of the first three vortices. After 1515 UT three more vortices passed, all moves eastward.

Figure 8 provides snapshots of the plasma flow in a large area of the high latitude ionosphere, extending $14^{\circ}$ in magnetic latitude and 3 hours in local time. We present 8 frames, each 1 min apart, during the passage of the three vortices that occurred between 1505 and 1512 UT. The equivalent drifts, deduced from the magnetic deflections of the Greenland magnetometers, are plotted as red vectors. We use longer vectors to represent larger magnetic fluctuations. The Sondrestrom LOS velocities are displayed using black or white arrows depending upon the sign of the velocity (toward or away the station). The LOS velocity from the Stokkseyri HF radar are depicted using green vectors. The large dot with the initial "St" indicates the location of the Stokkseyri HF radar; and the location of the magnetometer stations are each labeled in the panel corre-

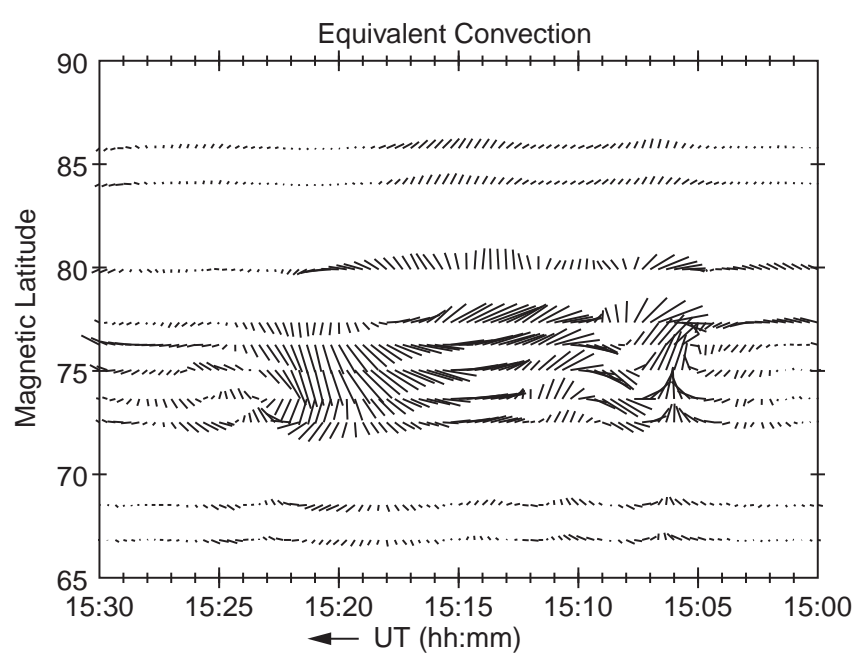

Fig. 7. Plot of the horizontal magnetic perturbation vectors corresponding to the variations of Fig. 5. The vectors have been rotated $90^{\circ}$ counterclockwise and plotted every $20 \mathrm{~s}$ during the time interval between 1500 and 1530 UT. Note the time scale increases from right to left in order to display the east-west extension of the vortices that were traveling eastward 

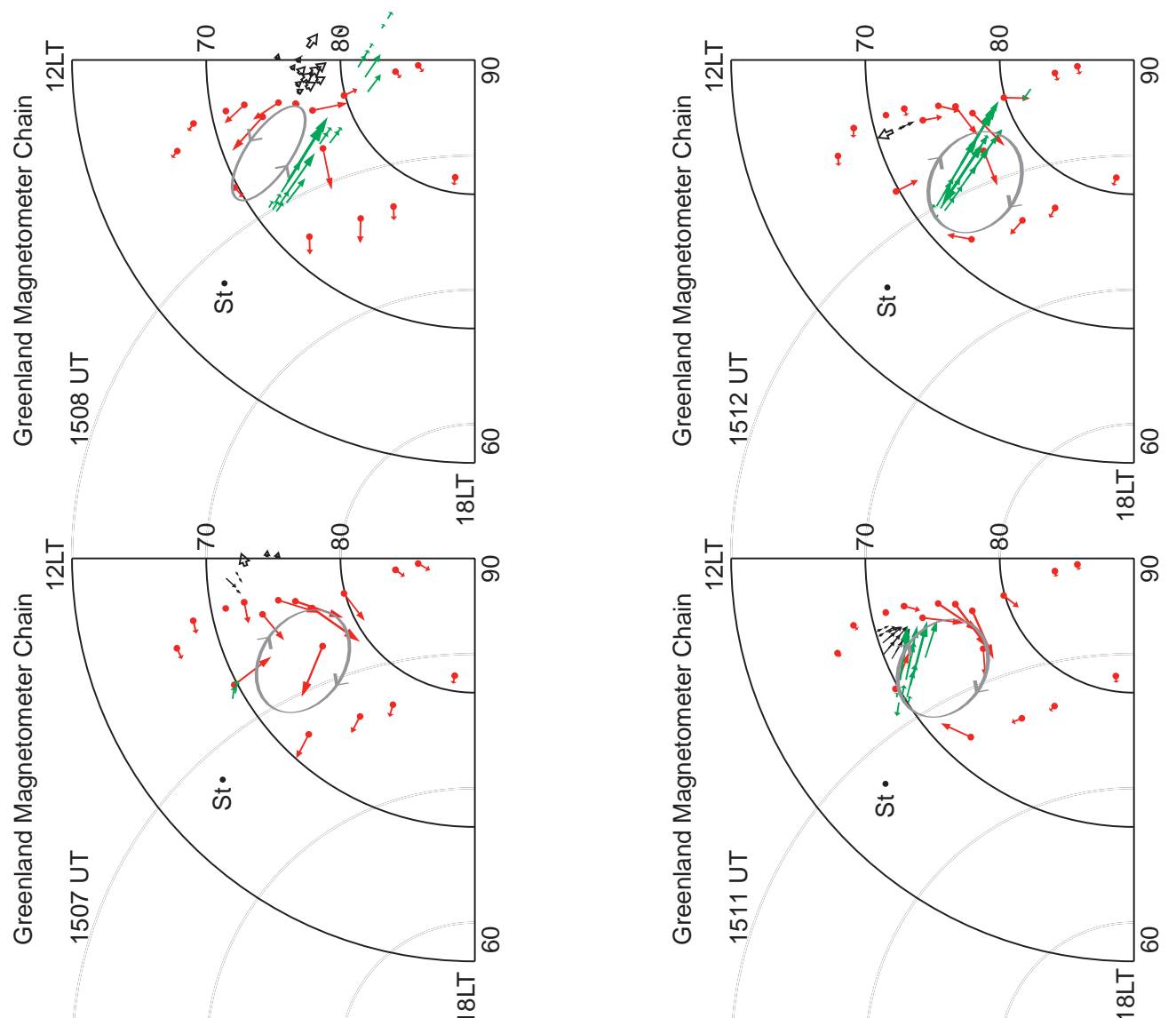

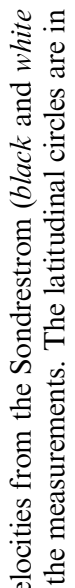
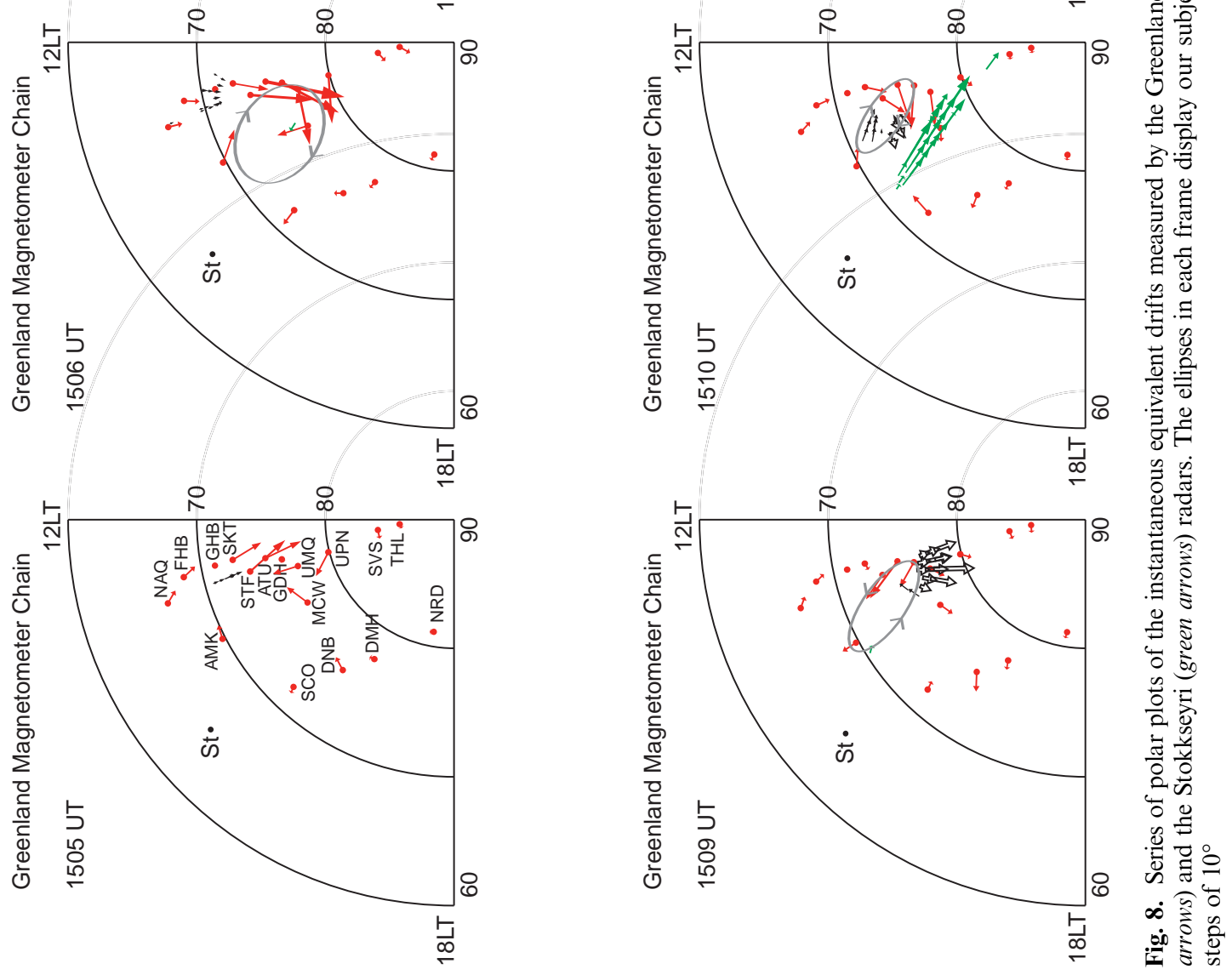


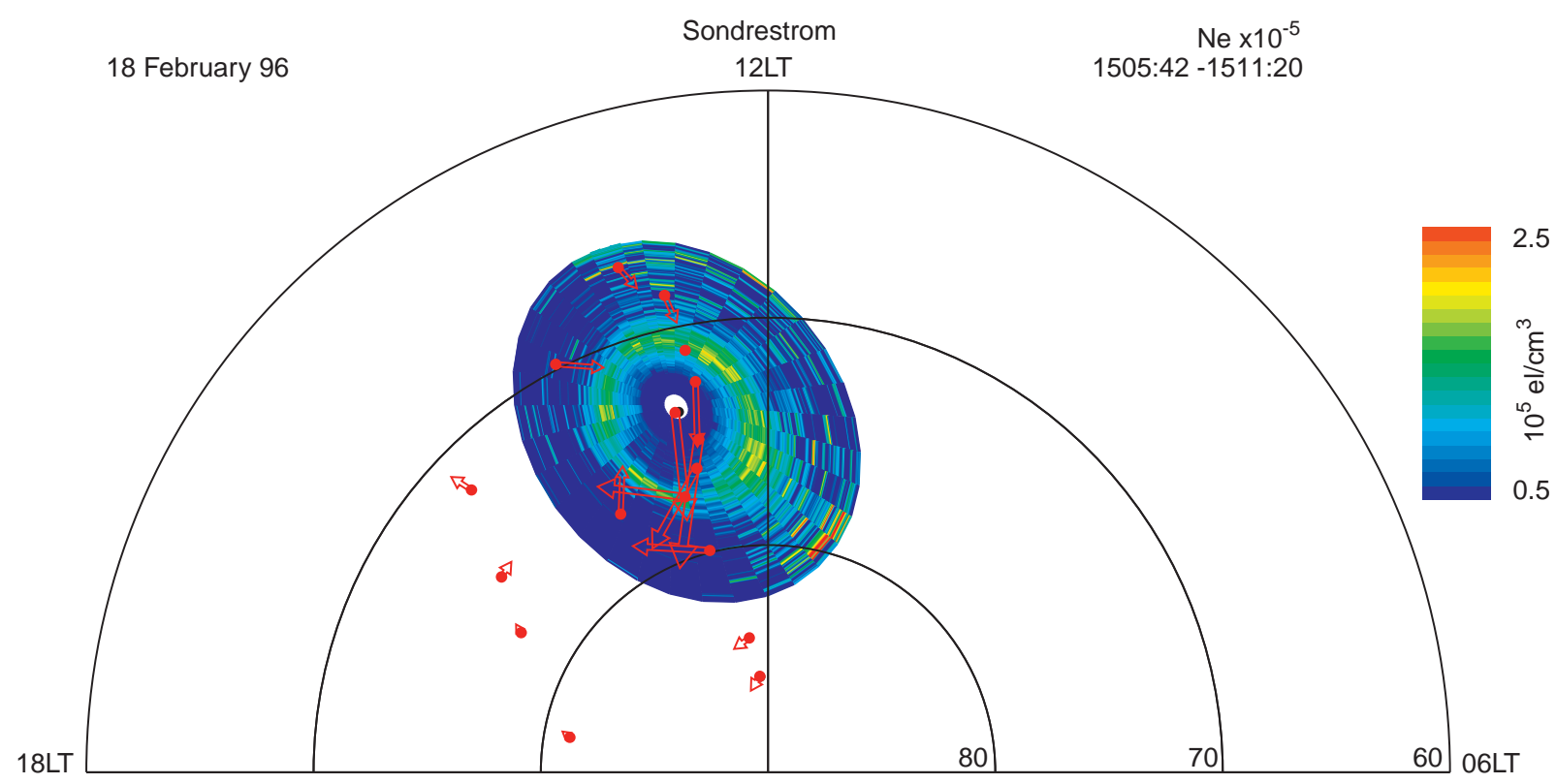

Fig. 9. Plot of the number density measured by the Sondrestrom radar between 1505:42 and 1511:20 UT. Superimposed in red arrows are the equivalent drifts measured by the magnetometers at $1506 \mathrm{UT}$.

sponding to 1505 UT. We have used only the afternoon quadrant of the $\mathrm{CG}$ coordinate system to display the vectors. The gray circles with arrows, as seen in some of the panels, denote our subjective interpretation of the location and size of the TCV associated with the observed plasma velocities. Each panel in Fig. 8 shows the equivalent drifts measured at the minute and the LOS velocities from the Sondrestrom and Stokkseyri radars gathered $\pm 20 \mathrm{~s}$ from the time indicated at the top left corner of each panel.

Figure 8 shows that at 1506 UT a vortex was centered at approximately $74^{\circ}$ magnetic latitude. This vortex is seen in later frames to move eastward and then fade as it moves away from the dayside at 1508 UT. The vortex observed at 1506 UT has a clockwise vorticity, which corresponds to a filamentary field aligned current directed away from the Earth. At 1508 UT, a second vortex appears with a counter-clockwise plasma motion and consequently a current polarity opposite to the previous vortex. This vortex is more elliptical and its reconstruction is in agreement the Stokkseyri LOS velocities (green vectors) in the part of the vortex of poleward flows. At 1510 UT, the third vortex appears centered at $72.5^{\circ}$ magnetic latitude. In the following two panels, this vortex expands poleward and becomes more circular. During the passage of the third vortex, the Sondrestrom ISR was scanning toward the east and south of the station (1510 UT). In the east sector, the radar detected small amplitude LOS velocities reversing sign. This is indicative that the radar was pointing nearly perpendicular to the vortex flow and in agreement with the vortex flow at both sides from the point of perpendicularity. When the radar is scanning to the south the LOS velocities are again consistent with a clockwise vortical motion. In general, it seems that the vortices have a rapid onset and probably last only few
Note the precise collocation of the region of large drifts and the F-region density depletion toward the north of the station

minutes during their transit across Greenland. In spite of this short lifetime the effect of the vortices on the density, as seen in Fig. 9, is very pronounced.

Figure 9 shows the number density of Fig. $6 \mathrm{~b}$ translated to a CG coordinate system. We have also superimposed the equivalent drifts measured by the magnetometers at 1506 UT. The radar scan started at 1505:42 UT, when the antenna was pointing toward magnetic south. The antenna scanned clockwise, reaching the $\mathrm{N}_{\mathrm{e}}$ depletion at 1508:50 UT. This depletion is collocated with strong poleward equivalent drifts. As shown in Fig. 7, this poleward flow associated with the vortex passage lasted from 1505:40 to 1507:00 UT. This time seems long enough to create a factor of 2 density depletion which remained almost unchanged for several minutes. (It was seen by the Sondrestrom ISR at 1508:50 UT.) The magnetic perturbations were the largest during the period under discussion here, exceeding $100 \mathrm{nT}$. We do not have a direct radar measurement of the velocities in the first vortex, but based on the LOS velocities measured by the Stokkseyri, we infer that they were probably of the order of $2.5 \mathrm{~km} / \mathrm{s}$. These large velocities were able to create a substantial frictional Joule heating and enhance the temperature dependent $\mathrm{O}^{+}$recombination coefficient. This enhancement of the loss rate is responsible for eroding the number density at the $\mathrm{F}$ peak from a background level of $1.5 \times 10^{5} \mathrm{~cm}^{-3}$ down to $0.7 \times 10^{5}$ $\mathrm{cm}^{-3}$, as observed in the northern part of Fig. $6 \mathrm{~b}$.

Fig. 10a-f. Number density, line-of-sight velocity and ion temperature measured by the Sondrestrom ISR on February 18, 1996, during the time interval from 1530:20 and 1544:11 UT. The location of the density trough is seen in both density plots extending in the east-west direction. Enhanced $V_{l o s}$ and $T_{i}$ are seen somewhat collocated with the $\mathrm{N}_{\mathrm{e}}$ depletions. See text for details 

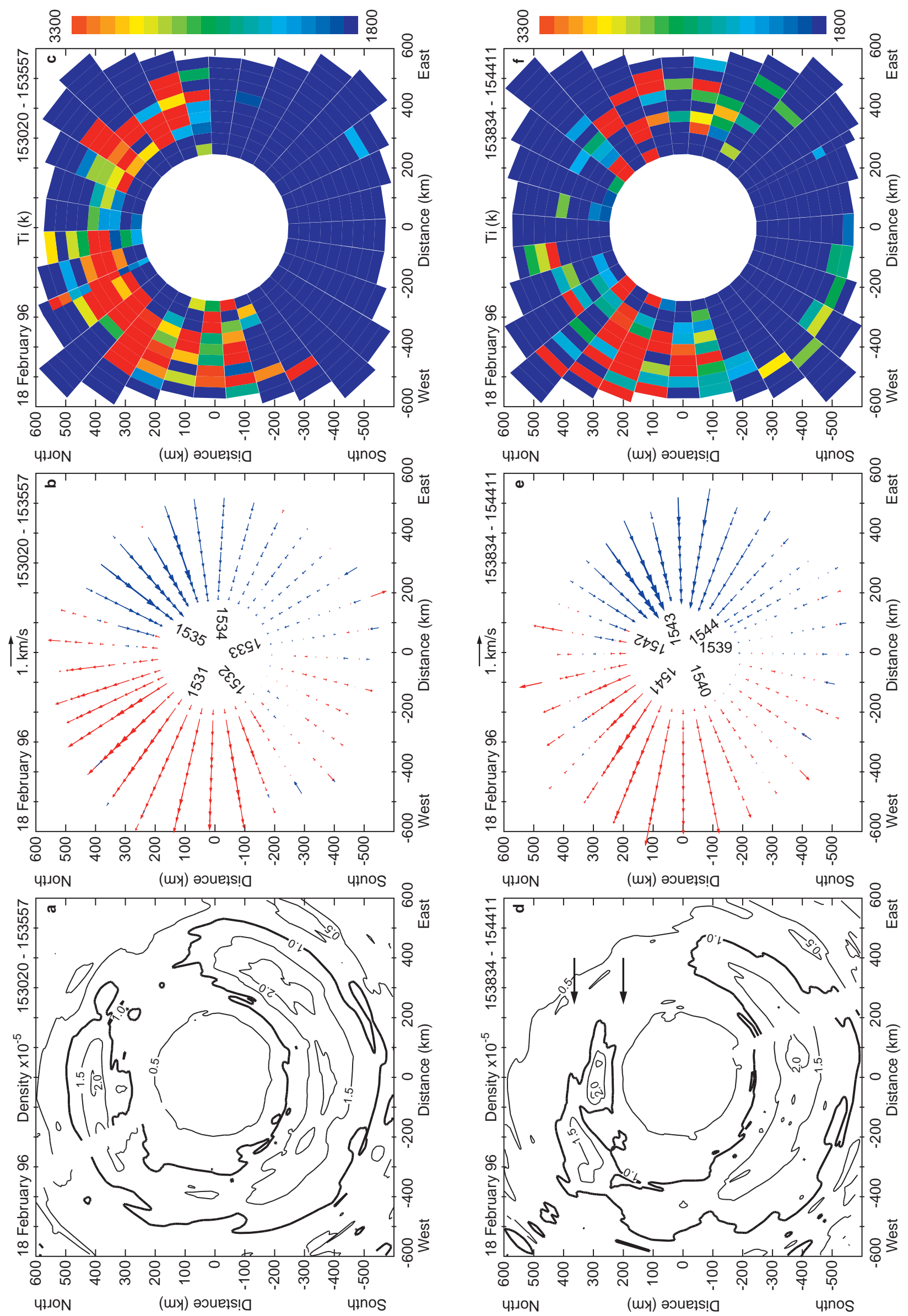


\section{Patch formation associated with large plasma flows}

After 1528 UT, the ground-based magnetometers did not detect the spiky magnetic deflections. In spite of this lower magnetic activity the Sondrestrom radar observed very pronounced density depletions and $\mathrm{N}_{\mathrm{e}}$ structures. In this section, we present the line-of-sight velocities from the SuperDARN, the Sondrestrom, and the EISCAT radars to imply that a different process, channels containing large plasma jets (LPJs), developed at high latitudes between 1530 and 1630 UT. Several LPJs were seen near the poleward boundary of the auroral oval during this time interval. The result of the occurrence of the first two LPJs was to form two sharp density depletions and a patch-like feature in between the density depletions.

\subsection{Sondrestrom ISR observations of LPJ}

Figure 10 presents the number density, line-of-sight velocity $\left(\mathrm{V}_{\mathrm{los}}\right)$, and the ion temperature measured by the Sondrestrom ISR during two consecutive AZ scans. The three panels at the top of Fig. 10 show the $\mathrm{N}_{\mathrm{e}}, \mathrm{V}_{\text {los }}$ and $\mathrm{T}_{\mathrm{i}}$ parameters observed during a $360^{\circ}$ counter-clockwise scan conducted between 1530:20 and 1535:57 UT. The three panels in the bottom row depict the same geophysical quantities but measured during a clockwise scan that started at 1538:34 and ended at 1544:11 UT. The times at the center of Fig. 10b, e indicate the exact hour and minute when the antenna was pointing at the precise azimuth angle where the numbers are placed.

The variability of the number density near the Sondrestrom station is registered in Fig. 10a. This figure shows that the deepest depletion $\left(0.5 \times 10^{5} \mathrm{~cm}^{-3}\right)$ is located between $40^{\circ}$ and $70^{\circ}$ clockwise azimuth from geographic north. A second density depletion, however less pronounced $\left(10^{5} \mathrm{~cm}^{-3}\right)$, is placed to the west of the station. Eight minutes later, Fig. 10d reveals that the deep depletion has evolved into a much wider extension, spanning from $35^{\circ}$ up to $90^{\circ}$ azimuth and aligned in the east-west direction. The poleward side of this depletion is indicated by arrow 1. A second depletion in Fig. 10d is indicated by arrow 2 . This latter channel is located to the north and $400 \mathrm{~km}$ away from the station had initially a density equal to $\sim 2 \times 10^{5} \mathrm{~cm}^{-3}$ (Fig. 10a) and has later decreased down to $0.5 \times 10^{5} \mathrm{~cm}^{-3}$ (Fig. 10d). The result of the formation of the $\mathrm{N}_{\mathrm{e}}$ depletion is to create a density feature bounded equatorward by the reduced densities mentioned, and poleward by another channel of low densities. Therefore, a narrow region, almost $100 \mathrm{~km}$ wide (in the north-south direction), and containing peak densities equal to $2.0 \times 10^{5} \mathrm{~cm}^{-3}$ was created almost detached from the auroral oval. This density feature is located upstream the sunward convection in the dusk cell, and probably in a region where the flow is sunward.

The location of the $T_{i}$ enhancements has also varied between the two scans. Figure $10 \mathrm{c}$ shows that $T_{i}$ is above the background value of $2000 \mathrm{~K}$ at the northern part of the scan. Peak values of $T_{i}$ are at $\sim 35^{\circ}$ and $-45^{\circ}$ azimuth. Figure 10f reveals that the $T_{i}$ enhancements have become more localized. They are restricted to 2 different areas of the scan, one at $55^{\circ}$ and the other at $-60^{\circ}$ azimuth angle. Careful comparison of the $\mathrm{N}_{\mathrm{e}}$ and $\mathrm{T}_{\mathrm{i}}$ plots for both scans indicates that the deep density depletions are not exactly collocated with the larger values of $T_{\mathrm{i}}$. This is more evident at the western part of both scans, and especially in the northwestern part of the first scan (see Fig. 10a), where $T_{i}$ is above $4000 \mathrm{~K}$ and there is no apparent $\mathrm{N}_{\mathrm{e}}$ depletion. This fact suggests that the erosion of the plasma density is an accumulative effect and the depth of the $\mathrm{N}_{\mathrm{e}}$ depletion depends not upon the instantaneous convection (or instantaneous $\mathrm{T}_{\mathrm{i}}$ enhancement), but instead upon the recent-time history (5-10 $\mathrm{min})$ of the velocity field and upon the value of the density at the point where the plasma originates. The discrepancy between the location of the $T_{i}$ enhancement channels and the $\mathrm{N}_{\mathrm{e}}$ depletions can be attributed to distribution of the gradients of the background density. The eastern depletion is fed by plasma residing at later local times, which is less dense than the plasma that feeds the western depletion. The following subsection presents maps of $\mathrm{V}_{\text {los }}$, gathered with the SuperDARN radars with a much higher resolution, to capture the evolution of the flows during the formation of the $\mathrm{N}_{\mathrm{e}}$ depletion.

\subsection{SuperDARN radar observations of $L P J$}

Figure 11 reproduces $\mathrm{V}_{\text {los }}$ measured with two of the SuperDARN coherent radars. Each frame covers only the postnoon-dusk quadrant and the magnetic latitude range between $60^{\circ}$ and $90^{\circ}$. The magnitude and sign of $\mathrm{V}_{\text {los }}$ have been color-coded to avoid cluttering the figures. Almost every panel of Fig. 11 shows values of $\mathrm{V}_{\text {los }}$ above $1 \mathrm{~km} / \mathrm{s}$ in a region near noon and extending between $73^{\circ}$ and $77^{\circ}$ magnetic latitude. The four panels at the top show $\mathrm{V}_{\text {los }}$ reaching $1500 \mathrm{~m} / \mathrm{s}$ in a region where the convective flows are mainly zonal and the direction of the line-of-sight from the Stokkseyri radar is probably almost parallel to this zonal flow. Poleward of the region of large flows $\left(>78^{\circ} \mathrm{mag}\right.$. latitude) the velocity decreases rapidly and becomes near $0 \mathrm{~m} / \mathrm{s}$ at $80^{\circ}$. Equatorward of the region of large flows, the Stokkseyri radar did not measure plasma flows probably due to the absence of density structures. We have superimposed on the plot that starts at 1532 UT a continuous trace that delineates the region where $\mathrm{V}_{\text {los }}$ is larger than $1 \mathrm{~km} / \mathrm{s}$. This contour line has also been duplicated in the following two panels to indicate how the location of large $\mathrm{V}_{\text {los }}$ changes in the two scans that followed 1534 UT. The high velocities in the panel that starts at 1536 UT are located a few degrees poleward from their original location at 1532 UT. It has moved very rapidly to this location. The channel of large $V_{\text {los }}$ seems to decay in the adjacent panel at $1538 \mathrm{UT}$, but it reappears $2 \mathrm{~min}$ later at 1540 UT near its prior more equatorial location. The LPJ remains stationary at this location until 1554 UT (not shown here) when it briefly moves back to a poleward location. It is worth mentioning that coherent 

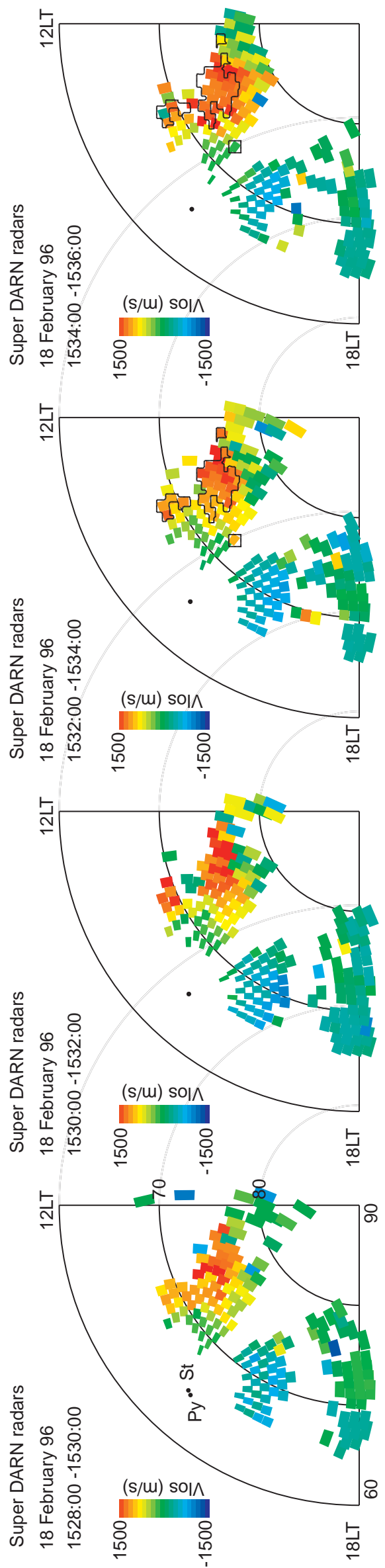
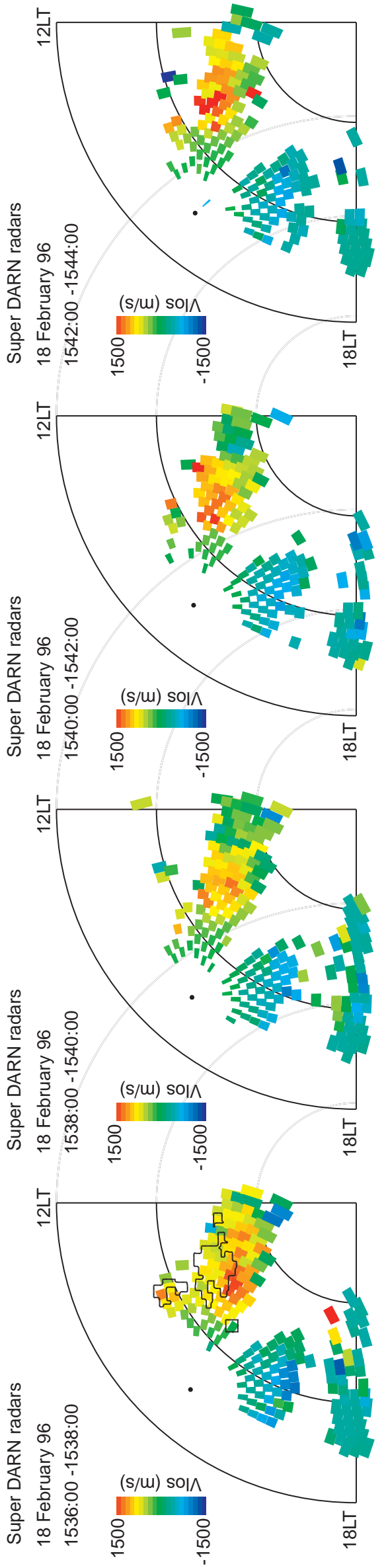

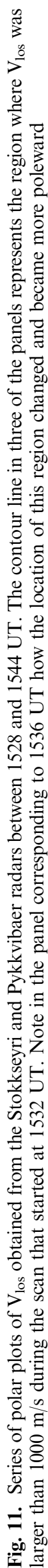


as well as incoherent radars are able to measure only one component of the velocity vector. Thus, it is quite possible that the radar has detected a large $\mathrm{V}_{\text {los }}$ due solely to a better alignment between the vector velocity and the line-of-sight radar direction. In the following subsection, we present resolved horizontal velocities, and demonstrate that the movement of the channel of large $\mathrm{V}_{\text {los }}$ toward higher latitudes corresponds to a phase motion of the LPJ and not to a realignment of the plasma velocity with the radar looking direction.

\subsection{Analysis of velocities associated with LPJ}

To emphasize that the variability of the location of the large plasma jets is a likely mechanism of patch formation, we have conducted a novel calculation of the vector velocities using $\mathrm{V}_{\text {los }}$ measurements conducted with the EISCAT, Sondrestrom, and SuperDARN radars and some information derived from statistical convection patterns. The algorithm that was used to resolve the velocities consisted of a multi-step process which can be defined in the following manner. First, we computed the horizontal velocity vector pairing $\mathrm{V}_{\text {los }}$ from the Stokkseyri and Sondrestrom radars only for cases when the probed volumes from these two radars were nearly collocated. We repeated the same procedure for the overlapping line-of-sight velocities measured with the Pykkvibaer and EISCAT radars. In this analysis only two components of the full vector velocity are calculated; the third component, the one along the magnetic field, is assumed to be zero (Valladares and Carlson, 1991). Results of this analysis are presented in Fig. 12a-d, where we show the horizontal velocities for four periods around the time of the poleward phase motion of the LPJ. Large westward flows, very typical of the near noon dusk cell, are seen in the first three panels. Figure $12 \mathrm{~d}$ shows a clockwise rotation of the flows near noon, also a characteristic of a smaller dusk cell in a typical 2-cell pattern.

Second, we performed a least square fitting analysis of several Heelis-type convection patterns (Heelis et al., 1982) to the horizontal velocities obtained in step 1 . Third, after the best fit was obtained, we used the information of the plasma flow direction in the selected convection pattern to determine the component of the velocity perpendicular to the line-of-sight in the SuperDARN observations. The resolved horizontal velocities for each measurement of $\mathrm{V}_{\text {los }}$ of the Stokkseyri and Pykkvibaer radars are presented in Fig. 12e-h.

Figure 12e shows the presence of a LPJ containing velocities above $2 \mathrm{~km} / \mathrm{s}$ and located between $72^{\circ}$ and $75^{\circ}$ magnetic latitude at $14 \mathrm{LT}$. The LPJ is embedded in a region of sunward convection, close to the location where the plasma convection turns to an antisunward orientation. The LPJ extends longitudinally up to 16 LT, however, with much reduced velocities of order $1 \mathrm{~km} / \mathrm{s}$ at the tail of the LPJ. These values are much lower than the values observed at 14 LT, but they are still bounded latitudinally by smaller velocities. At 1534 UT, the LPJ has shifted to higher latitudes but the maximum values are slightly less pronounced. Two minutes later the LPJ intensifies, and moves poleward, extending between $75^{\circ}$ and $77^{\circ}$ magnetic latitude (note the dotted line is traced at $75^{\circ}$ ). Simultaneously, there exists a reduction in the size of the polar cap. At 1542 UT (not shown here) the LPJ returns to its previous more equatorward location and stays at this magnetic latitude for $10 \mathrm{~min}$, before moving poleward again.

Figure 12 does not show any of the SuperDARN V $\mathrm{V}_{\text {los }}$ data measured in the dawn cell. At this local time the dawn cell is located westward of the Sondrestrom site. Measurements conducted with the Saskatoon and Kapuskasing radars in the morning sector did not present any signature of LPJs. These radars observed only modest line-of-sight velocities of order $500 \mathrm{~m} / \mathrm{s}$.

\section{Discussion}

Several mechanisms have been previously suggested to explain the formation and transit of the polar cap patches. Tsunoda (1988), in his extended review paper, outlined the most important processes conducive for patch formation. He stressed the importance of continuous changes in the sign of the IMF $B_{y}$ or $B_{z}$ components and/or the localized production of plasma density in the cusp region as prime candidates for patch formation. Recent work undertaken by Sojka et al. $(1993,1994)$ have demonstrated that in fact the $B_{y}$ switching mechanism not only generates isolated density enhancements inside the polar cap, but equally important it reproduces the seasonal and UT morphology of the polar cap patches seen at the center of the polar cap (Buchau et al., 1983). The net effect of a $B_{y}$ flip can be understood as a redirection of the elongated TOI toward different locations within the polar cap. Repetitive flips of $B_{y}$ will successively chop the otherwise continuous TOI. A more detailed comparison between the occurrence of scintillations near the center of the polar cap and the morphology of the TOI density, when it transits across the polar cap, has revealed a remarkable similarity between their seasonal and UT dependencies (Basu et al., 1995). This agreement endorses the fact that the TOI is the source of the density enhancements within the patches. However, in spite of the success of the $B_{y}$ switching mechanism, statistics of the switching rate of $B_{y}$ do not conform with the rate that patches are seen transiting the polar cap (10-20 min). Moreover, any patch formation mechanism that uses the TOI as the source of the enhanced plasma will have a UT and a seasonal statistics very similar to the ones described by Sojka et al. (1994). Therefore, this new mechanism will be in agreement with the statistics of scintillations at the center of the polar cap (Basu et al., 1995).

Another mechanism of patch formation that has been studied recently is the IMF $B_{z}$ switching mechanism described by Valladares et al. (1998). However, this mechanism may only produce a single polar cap patch shortly after $B_{z}$ turns negative. Unless an additional mechanism is invoked, a single $B_{z}$ flip will not reproduce the most salient feature of polar cap patches which is the 

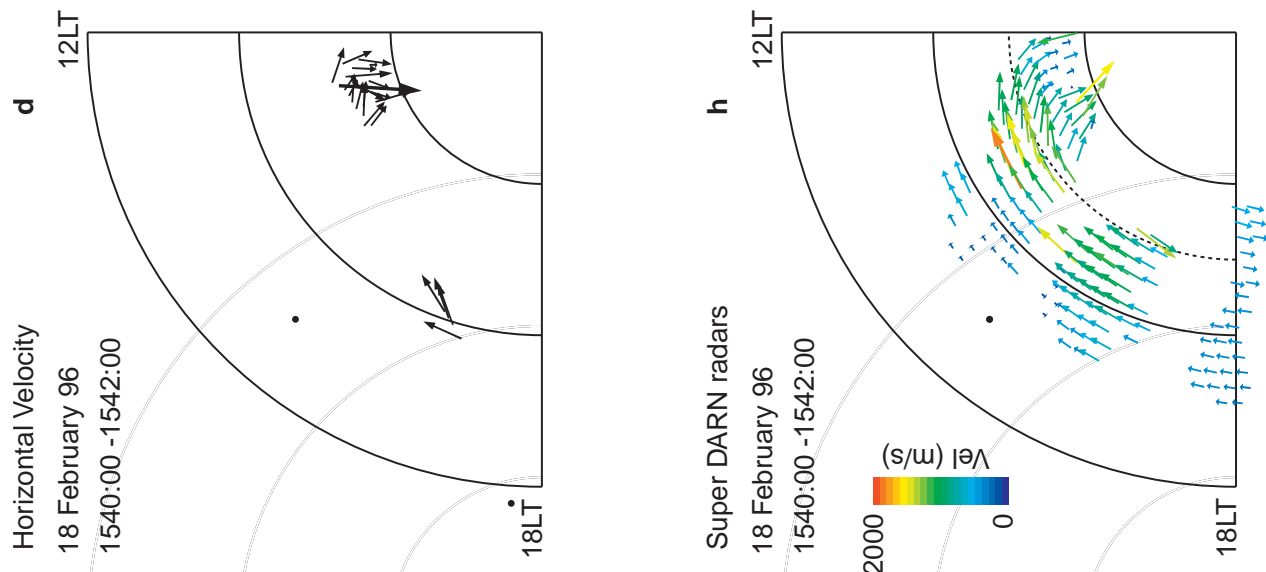

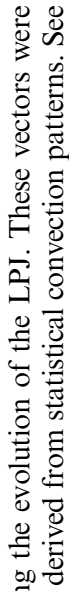
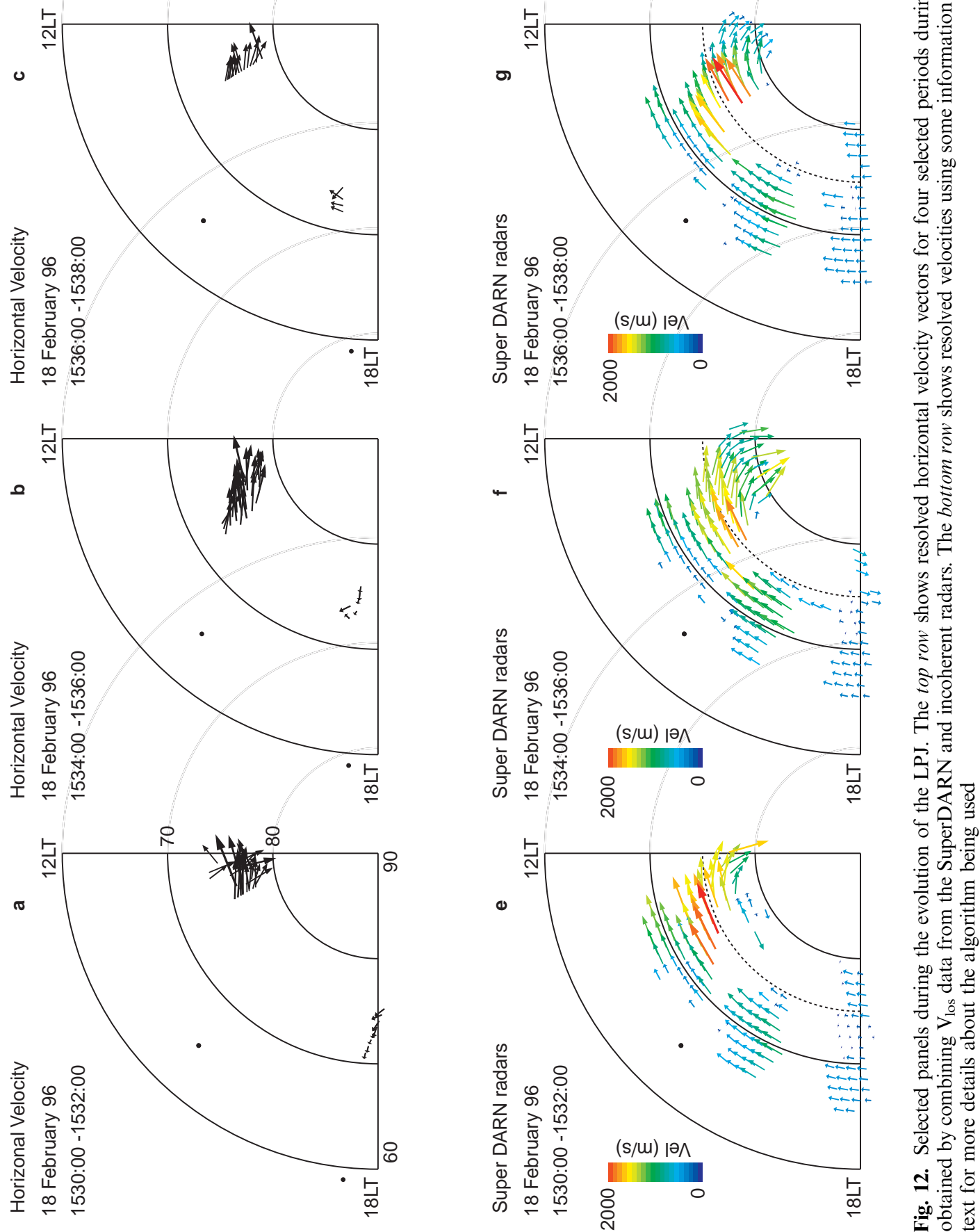
repetitiveness of the patches when they transit the polar cap. During IMF $B_{z}$ southward conditions, we typically observe, inside the polar cap, a train of several patches and rarely a single patch (Weber et al., 1991; Fukui et al., 1994).

In a different approach, Rodger et al. (1994), and Valladares et al. (1994) suggested that flow channel events or large plasma jets can produce enhanced losses creating a region of reduced number densities across the TOI. Rodger et al. (1994) suggested that a patch could be formed by the combined effect imposed by the presence of a channel containing large plasma flow and a reorganization of the convection pattern caused by changes in the IMF $B_{y}$ component.

In this work, we put forth evidence for two different mechanisms that are able to generate large-scale $(>100 \mathrm{~km}$ ) density structures acting near the poleward boundary of the oval during $B_{z}$ south conditions. We also indicate the manner in which the background convection can carry the newly formed density structures toward and into the polar cap.

The first patch formation mechanism described deals with the passage of a train of TCVs through the dayside ionosphere to produce channels embracing lower densities. After the vortices have passed, the density structure will be convected into the polar cap by the dayside flow velocity of a typical $B_{z}$-south 2-cell convection pattern. Figure 13 presents our conceptual interpretation of how a patch-like structure can be formed in the dayside region. The large flow velocity residing at the interface between two adjacent vortices will produce an enhanced loss of the plasma density (see dotted area in Fig. 13). This region of reduced number density will rotate, following the vortex outer radius isolating a region of dayside number density. After the vortex has moved outside the region of depleted densities, the region of low density will start to fill up by solar ionization. However, this refilling process could well take up to tens of minutes. During this time, polar antisunward convection will transport the newly formed

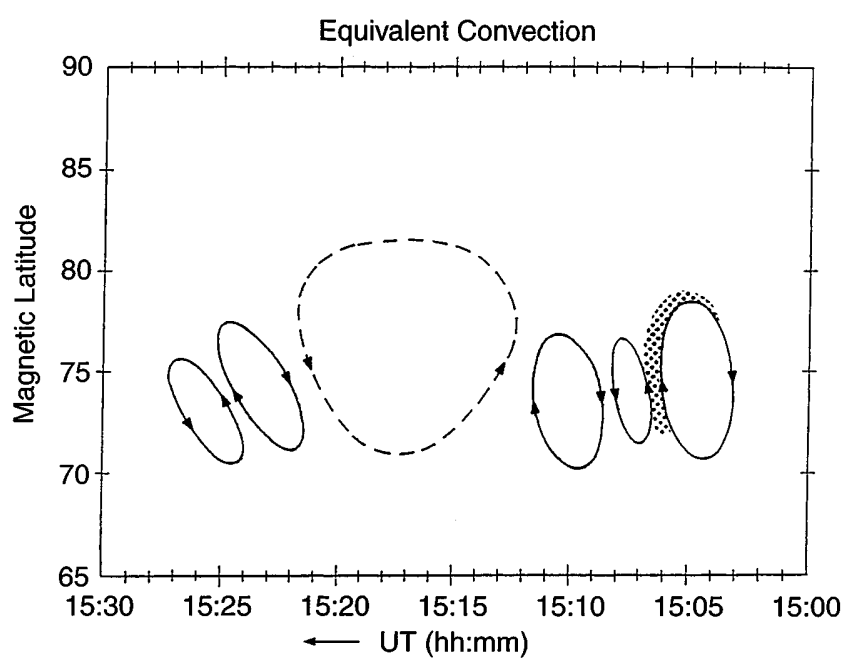

Fig. 13. Schematic drawing showing the transit of the TCVs and the density depletion formed in between the regions of large flow patch-like structure into and toward the center of the polar cap. Note that in this scenario the patch will be a close image of the vortex.

On February 18, 1996, a series of six vortices were reconstructed based on the equivalent magnetometer drifts (Fig. 7) and the instantaneous flows of Fig. 8. Thus, it is also likely the flows associated with any of the five successive vortices have distorted the original poleward aligned density depletion by stirring the plasma. In this picture, further breaking, splitting, and tearing of the patch-like structure into smaller patch-like structures may have occurred later. This second-step structuring may explain the appearance of the density feature, seen by the Sondrestrom radar $450 \mathrm{~km}$ north of the station, during the scan that started at 1513:55 UT (Fig. 6c, d).

The first intensification of the polar cap potential in Fig. 4 was associated with the transit of the first vortex which contained the largest velocities of order $2.5 \mathrm{~km} / \mathrm{s}$ (measured by the Stokkseyri radar). The other remaining five vortices, although clearly detected by the magnetometers, were not associated with very large flows and did not seem to carve out detectable density depletions.

As postulated before, we believe that the density depletion was probably carved out by enhanced $\mathrm{O}+$ recombination. However, we recognize the possible role of plasma evacuation in forming an auroral ionospheric cavity (Doe et al., 1993) and removing part of the ionospheric electrons as they become charge carriers for a downward field-aligned current (Doe et al., 1995). Note that the second vortex, observed at 1507 UT and adjacent to the region of depleted densities, has a counter-clockwise rotational flow which indeed corresponds to downward directed current. Modeling work is needed to assess the importance of plasma evacuation and/or enhanced loss rate in producing the density depletions observed by the Sondrestrom ISR.

The second intensification of the cross polar cap potential occurred at 1530 UT (Fig. 4). We believe this is associated with the excitation of the LPJ seen in Fig. 12e. The LPJ becomes weaker and narrower at later local times; at the same time the polar cap potential decreases to values near $70 \mathrm{kV}$. This second mechanism that created large-scale structures on February 18, 1996, involves large plasma jets, but incorporates new, key information provided by the SuperDARN radars. The LPJs are not stationary but instead are affected by a periodic latitudinal phase motion. In general terms, LPJs are very efficient in creating regions of low plasma by bringing less dense plasma from earlier or later local times. They may require only few minutes to attain deep depletions (Valladares et al., 1996). The large plasma velocity of the LPJ enhances the $\mathrm{O}+$ recombination loss and due to its large longitudinal extension, much less dense plasma can be carried from later local times. This mechanism is different from the mechanism suggested by Rodger et al. (1994) which depended on a timely variation of the $B_{y}$ component. Although in other cases the poleward motion of the LPJ could be attributed to variations of the IMF $B_{y}$ component, measurements of 


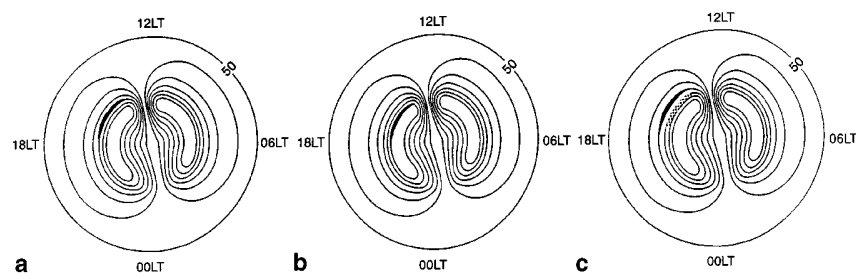

Fig. 14a-c. Schematic representation of the evolution of the LPJ and its possible effect for the formation of an isolated density structure bounded by two regions of recently depleted densities

the IMF carried out by the WIND satellite at the time of interest (14-15 UT) registered only mild variations of $B_{y}$ (between 0 and $-1 \mathrm{nT}$ ).

The two azimuth scans of Fig. 10 display the formation of a density structure bounded by two channels of reduced densities (see arrows in Fig. 10d). This density structure was observed by the Sondrestrom radar approximately at 1542 UT. Both low-density channels coincide with the location of the two LPJs that were seen in the SuperDARN data of Fig. 12e and g.

Figure 14 illustrates our concept on how the LPJ phase motion developed. It is initially located at $73.5^{\circ}$ magnetic latitude and then changes to $77^{\circ}$ magnetic latitude a few minutes later. A region of dayside density, represented by the shaded area of Fig. 14c, is confined by two regions of low density, one poleward and another equatorward. After the region of dayside density is isolated from the rest of the oval densities, it will be carried by the antisunward plasma convection and enter the polar cap, thus initiating its transit toward the nightside of the polar cap.

Whalen (1989) presented number densities measured during the IGY, and characterized the daytime F layer trough under conditions of solar maximum, winter solstice. He postulated that the afternoon trough is generally continuous and stationary for a duration of many hours in a magnetic latitude/magnetic local time coordinate system. We have found a density depletion located between $73^{\circ}$ and $77^{\circ}$ magnetic latitude that forms and persists after $B_{z}$ turns south containing some characteristics very similar to the trough described by Whalen (1989). In addition, we have confirmed that it is collocated with channels containing large flows (or LPJs) that extended for thousands of kilometers (Fig. 12e-g). The SuperDARN radars complete a scan every two min and this high resolution data has revealed a new important feature which is instrumental for the formation of the polar cap patches. There exists a phase motion of the LPJ which occurs on a time scale of 10 20 min. This cyclical motion of the LPJ may create the sequence of patches that are commonly detected within the polar cap (Buchau et al., 1983).

\section{Conclusions}

This study has led to the following conclusions:

1. The Greenland chain of magnetometers in conjunction with the Sondrestrom and Stokkseyri radars observed the transit of a train of vortices moving eastward across Greenland. Up to six vortices were identified in the magnetometer data. They were moving with a velocity equal to $5.7 \mathrm{~km} / \mathrm{s}$.

2. The Sondrestrom ISR confirmed the formation of a density depletion closely collocated with a region where very intense equivalent plasma drifts were seen 2 min earlier. The plasma erosion was caused by an enhancement of the $T_{i}$ dependent recombination rate of $\mathrm{O}^{+}$augmented by Joule frictional heat. We speculate that the circular motion of the plasma inside the vortex may create an elliptical torus of reduced density. This region of low density may convect toward and across the polar cap following the typical $B_{z}$ south convection pattern which dominated during the observations.

3. The EISCAT, the Sondrestrom, and the SuperDARN radars detected the width, longitudinal extension, and temporal evolution of a large plasma jet that appeared in the dayside sector of the dusk cell a few minutes after the TCVs were extinct. The LPJs were not stationary. They moved poleward, faded, and then reappeared in a matter of minutes.

4. A factor of 4 density depletion and ion temperatures above $4000 \mathrm{~K}$ were seen by the Sondrestrom ISR sometimes coinciding with the location of the LPJ.

5. The latitudinal poleward motion of the LPJ created an elongated density structure bounded by two regions of low-density plasma. This almost isolated density structure is seen in Fig. 10d and was scanned by the Sondrestrom radar at 1542 UT. Two regions, indicated by arrows in Fig. 10d, were previously eroded; however, at different times by two LPJs located at different latitudes. Important contributors to the formation of the regions of low density are the time-history of the plasma flow and the gradient in the background daytime ionosphere.

6. Another poleward motion of the LPJ was observed 18 min later, suggesting that this mechanism of patch formation may indeed produce a sequence of density features separated by 10-20 min.

Acknowledgements. We thank Peter Stauning of the Danish Meteorological Institute for making the magnetometer data available for this study, J. Kelly and M. McCready for providing the Sondrestrom radar data in raw format, R.P. Lin for providing us the WIND 3DP plasma observations and K. Fukui for drafting Fig. 13 and 14. We acknowledge the valuable effort provided by P. Knoop of the University of Michigan for installing the UARC system at the EISCAT site, and T. Pedersen of USU for traveling to Sondrestrom. The work at Boston College was partially supported by NSF grants INT-9603362, ATM-9404088, and ATM-9613925, and by Air Force Research Laboratory contracts F19628-97-C-0094, and in Toulouse under CNRS Grant 97N92/0157. JVR was supported during the February 1996 campaign by a National Research Council-AFOSR Research Associateship and by travel funds from the Phillips Laboratory Geophysics Directorate. The analysis of the SuperDARN data was supported by NSF Grant ATM-9502993. Support for the operation of the Super DARN radars used in this study was provided by funding agencies in Canada, France, the UK, and the USA. We thank the Danish Commission for Scientific Research 
in Greenland for permission to conduct ground experiments at Sondrestrom under continuing project A16-91. EISCAT is an International Scientific Association supported by the Research Councils of Finland (SA), France (CNRS), Germany (MPG), Japan (NIPR), Norway (NAVF), Sweden (NFR) and the United Kingdom (PPARC).

The Editor in Chief thanks J. Scali for his help in evaluating this paper.

\section{References}

Baker, K. B., and S. Wing, A new magnetic coordinate system for conjugate studies of high latitudes, J. Geophys. Res., 94, 9139, 1989.

Basu, S., Su. Basu, J. J. Sojka, R. W. Schunk, and E. MacKenzie, Macroscale modeling and mesoscale observations of plasma density structures in the polar cap, Geophys. Res Lett., 22, 881, 1995.

Berkey, T., L. L. Cogger, S. Ismail, and Y. Kamidi, Evidence for a correlation between sun-aligned arcs and the interplanetary magnetic field direction, Geophys. Res. Lett., 3, $145,1976$.

Buchau, J., and B. W. Reinisch, Electron density structures in the polar F region, Adv. Space Res., 11, (10)29, 1991.

Buchau, J., B. W. Reinisch, E. J. Weber, and J. G. Moore, Structure and dynamics of the winter polar cap F region, Radio Sci., 18, 995, 1983.

Buchau, J., E. J. Weber, D. N. Anderson, H. C. Carlson, Jr., J. G. Moore, B. W. Reinisch, and R. C. Livingston, Ionospheric structures in the polar cap: their origin and relation to 250MHz scintillation, Radio Sci., 20, 325, 1985.

Clauer, C. R., and A. J. Ridley, Ionospheric observations of magnetospheric low-latitude boundary layer waves on August 4, 1991, J. Geophys. Res., 100, 21873, 1995.

Cowley, S. W. H., J. P. Morelli, and M. Lockwood, Dependence of convection flows and particle precipitation in the highlatitude dayside ionosphere on the $X$ and $Y$ components of the interplanetary magnetic field, J. Geophys. Res., 96, 5557, 1991.

Crooker, N. U., G. L. Siscoe, C. T. Russell, and E. J. Smith, Factors controlling degree of correlation between ISEE 1 and ISEE 3 interplanetary magnetic field measurements, J. Geophys. Res., 87, 2224, 1982.

Crowley, G., Critical review on ionospheric patches and blobs, in The Review of Radio Science 1992-1996, Oxford University Press, 1996.

de la Beaujardiere, O., J. D. Craven, V. B. Wickwar, G. Candal, J. M. Holt, L. A. Frank, L. H. Brace, D. S. Evans, and J. D. Winningham, Universal time dependence of nighttime $\mathrm{F}$ region densities at high latitudes, J. Geophys. Res., 90, 4319, 1985.

Doe, R. A., M. Mendillo, J. F. Vickrey, L. Zanetti, and R. Eastes, Observations of nightside auroral cavities, J. Geophys. Res., 98, 293, 1993.

Doe, R. A., J. F. Vickrey, and M. Mendillo, Electrodynamic model for the formation of auroral ionospheric cavities, J. Geophys. Res., 100, 9683, 1995.

Foster, J. C., and J. R. Doupnik, Plasma convection in the vicinity of the dayside cleft, J. Geophys. Res., 89, 9107, 1984.

Friis-Christensen, E., M. A. McHenry, C. R. Clauer, and S. Vennerstrom, Ionospheric traveling convection vortices observed near the polar cleft: a triggered response to sudden changes in the solar wind, Geophys. Res. Lett., 15, 253, 1988.

Fukui, K., J. Buchau, and C. E. Valladares, Convection of polar cap patches observed at Qaanaaq, Greenland during the winter of 1989-1990, Radio Sci., 29, 231, 1994.

Greenwald, R. A., et al., DARN/SuperDARN: a global view of the dynamics of high-latitude convection, Space Sci. Rev., 71, 761, 1995.
Heelis, R. A., J. K. Lowell, and R. W. Spiro, A model of the highlatitude ionospheric convection pattern, J. Geophys. Res., 87, 6339, 1982.

Hill, J. R., Sudden enhancements of F layer ionization in polar regions, J. Atmos. Sci., 20, 492, 1963.

Ismail, S., D. D. Wallis, and L. L. Cogger, Characteristics of polar cap sun-aligned arcs, J. Geophys. Res., 82, 4741, 1977.

Kelly, J. D., and J. F. Vickrey, F region ionospheric structure associated with antisunward flow near the dayside cusp, Geophys. Res. Lett., 11, 907, 1984.

Kelly, T. J., N. U. Crooker, G. L. Siscoe, C. T. Russell, and E. J. Smith, On the use of a sunward libration-point-orbiting spacecraft as an interplanetary magnetic field monitor for magnetospheric studies, J. Geophys. Res., 91, 5629, 1986.

Lassen, K., On the classification of high latitude auroras, Geofys. Publ., 29, 87, 1972.

Lockwood, M., P. E. Sandholt, S. W. H. Cowley, and T. Oguti, Interplanetary magnetic field control of dayside auroral activity and the transfer of momentum across the dayside magnetopause, Planet. Space Sci., 11, 1347, 1989.

Lockwood, M., P. E. Sandholt, A. D. Farmer, S. W. H. Cowley, B. Lybekk, and V. N. Davda, Auroral and plasma flow transients at magnetic noon, Planet. Space Sci., 38, 973, 1990.

McHenry, M. A., C. R. Clauer, and E. Friis-Christensen, Relationship of solar wind parameters to continuous dayside, high latitude, traveling ionospheric vortices, J. Geophys. Res., 95, 15007, 1990.

Rodger, A. S., and A. C. Graham, Diurnal and seasonal occurrence of polar cap patches, Ann. Geophysicae, 14, 533, 1996.

Rodger, A. S., M. Pinnock, J. R. Dudeney, K. B. Baker, and R. A. Greenwald, A new mechanism for polar patch formation, J. Geophys. Res., 99, 6425, 1994.

Ruohoniemi, J. M., R. A. Greenwald, K. B. Baker, J.-P. Villain, and M. A. McCready, Drift motions of small-scale irregularities in the high latitude F-region: an experimental comparison with plasma drift motions, J. Geophys. Res., 92, 4553, 1987.

Sandholt, P. E., M. Lockwood, T. Oguti, S. W. H. Cowley, K. S. C. Freeman, B. Lybekk, A. Egeland, and D. M. Willis, Midday auroral breakup events and related energy and momentum transfer from the magnetosheath, J. Geophys. Res., 95, 1039, 1990.

Sato, T., and G. F. Rourke, F-region enhancements in the Antarctic, J. Geophys. Res., 69, 4591, 1964.

Sojka, J. J., M. D. Bowline, R. W. Schunk, D. T. Decker, C. E. Valladares, R. Sheehan, D. N. Anderson, and R. A. Heelis, Modeling polar cap $F$ region patches using time varying convection, Geophys. Res. Lett., 20, 1783, 1993.

Sojka, J. J., M. D. Bowline, and R. W. Schunk, Patches in the polar ionosphere: UT and seasonal dependence, J. Geophys. Res., 99, 14 959, 1994.

Tsunoda, R. T., High-latitude F region irregularities: a review and synthesis, Rev. Geophys., 26, 719, 1988.

Valladares C. E., and H. C. Carlson Jr., The electrodynamic, thermal, and energetic character of intense sun-aligned arcs in the polar cap, J. Geophys. Res., 96, 1379, 1991.

Valladares, C. E., Su. Basu, J. Buchau, and E. Friis-Christensen, Experimental evidence for the formation and entry of patches into the polar cap, Radio Sci., 29, 167, 1994.

Valladares, C. E., D. T. Decker, R. Sheehan, and D. N. Anderson, Modeling the formation of polar cap patches using large plasma flows, Radio Sci., 31, 573, 1996.

Valladares, C. E., D. T. Decker, R. Sheehan, D. N. Anderson, T. Bullett, and B. W. Reinisch, Formation of polar cap patches associated with north-to-south transitions of the interplanetary magnetic field, J. Geophys. Res., in press, 1998.

Whalen, J., The daytime $\mathrm{F}$ layer trough and its relation to ionospheric-magnetospheric convection, J. Geophys. Res., 94, 17169,1989

Weber, E. J., and J. Buchau, Polar cap F-layer auroras, Geophys. Res. Lett., 8, 125, 1981. 
Weber, E. J., J. A. Klobuchar, J. Buchau, H. C. Carlson, Jr., R. C. Livingston O. de la Beaujardiere, M. McCready, J. G. Moore, and G. J. Bishop, Polar cap F layer patches: structure and dynamics, J. Geophys. Res., 91, 121, 1986.
Weber, E. J., J. F. Vickrey, H. Gallagher, L. Weiss, C. J. Heinselman, R. A. Heelis, and M. C. Kelley, Coordinated radar and optical measurements of stable auroral arcs at the polar cap boundary, J. Geophys. Res., 96, 17 847, 1991. 